\title{
Identification of the Functional Binding Site for the Convulsant Tetramethylenedisulfotetramine in the Pore of the $\alpha_{2} \beta_{3} \gamma_{2}$ $\mathrm{GABA}_{\mathrm{A}}$ Receptor $\$$
}

\author{
Brandon Pressly, Ruth D. Lee, Bogdan Barnych, Bruce D. Hammock, and Heike Wulff \\ Departments of Pharmacology (B.P., R.D.L, H.W.) and Entomology and Nematology, and Comprehensive Cancer Center (B.B., \\ B.D.H.), University of California, Davis, California
}

Received June 11, 2020; accepted October 6, 2020

\section{ABSTRACT}

Tetramethylenedisulfotetramine (TETS) is a so-called "caged" convulsant that is responsible for thousands of accidental and malicious poisonings. Similar to the widely used GABA receptor type $A\left(G_{A B A}\right)$ antagonist picrotoxinin, TETS has been proposed to bind to the noncompetitive antagonist (NCA) site in the pore of the receptor channel. However, the TETS binding site has never been experimentally mapped, and we here set out to gain atomistic level insights into how TETS inhibits the human $\alpha_{2} \beta_{3} \gamma_{2}$ $\mathrm{GABA}_{\mathrm{A}}$ receptor. Using the Rosetta molecular modeling suite, we generated three homology models of the $\alpha_{2} \beta_{3} \gamma_{2}$ receptor in the open, desensitized, and closed/resting state. Three different ligand-docking algorithms (RosettaLigand, Glide, and Swissdock) identified two possible TETS binding sites in the channel pore. Using a combination of site-directed mutagenesis, electrophysiology, and modeling to probe both sites, we demonstrate that TETS binds at the T6' ring in the closed/resting-state model, in which it shows perfect space complementarity and forms hydrogen bonds or makes hydrophobic interactions with all five pore-lining threonine residues of the pentameric receptor. Mutating T6' in either the $\alpha_{2}$ or $\beta_{3}$ subunit reduces the $\mathrm{IC}_{50}$ of TETS by $\sim 700$-fold in whole-cell patch-clamp experiments. TETS is thus interacting at the NCA site in the pore of the GABA receptor at a location that is overlapping but not identical to the picrotoxinin binding site.

\section{SIGNIFICANCE STATEMENT}

Our study identifies the binding site of the highly toxic convulsant tetramethylenedisulfotetramine (TETS), which is classified as a threat agent by the World Health Organization. Using a combination of homology protein modeling, ligand docking, site-directed mutagenesis, and electrophysiology, we show that TETS is binding in the pore of the $\alpha_{2} \beta_{3} \gamma_{2}$ GABA receptor type A receptor at the socalled T6' ring, wherein five threonine residues line the permeation pathway of the pentameric receptor channel.

\section{Introduction}

GABA receptor type $A\left(\mathrm{GABA}_{\mathrm{A}}\right)$ receptors are pentameric ligand-gated chloride channels (Olsen and Sieghart, 2008) that are activated by GABA, the major inhibitory neurotransmitter in the mammalian and insect nervous system. GABA receptors constitute the targets of many widely used drugs as well as of major household and agricultural pesticides. $\mathrm{GABA}_{\mathrm{A}}$ receptor agonists like barbiturates or positive allosteric modulators, as exemplified by the various typical and atypical benzodiazepines, reduce neuronal excitability and are widely

This work was supported by the CounterACT Program at National Institutes of Health Office of the Director with National Institute of Neurological Disorders and Stroke [Grant U54-NS079202] and [Grant R21NS110647]. There was also support from the National Institute of General Medical Sciences-funded Pharmacology Training Program [Grant T32GM099608] (to B.P.) and partial support from the National Institute of Environmental Health Sciences' Revolutionizing Innovative, Visionary Environmental Health Research (RIVER) [Grant R35ES030443] (to B.D.H.).

https://doi.org/10.1124/molpharm.120.000090.

S This article has supplemental material available at molpharm. aspetjournals.org. used as anesthetics, anxiolytics, and anticonvulsants (Krall et al., 2015; Olsen, 2015). In contrast, compounds that inhibit $\mathrm{GABA}_{\mathrm{A}}$ receptor functions increase neuronal firing. Depending on their species specificity, $\mathrm{GABA}_{\mathrm{A}}$ antagonists accordingly either constitute useful insecticides like fipronil or afoxalaner (Casida and Durkin, 2015), or, if they inhibit mammalian $\mathrm{GABA}_{\mathrm{A}}$ receptors like picrotoxinin or pentylenetetrazol, induce seizures and, at higher concentrations, death.

The extremely toxic tetramethylenedisulfotetramine (TETS) has been known since the early 1940s and was initially used as an antimold agent for furniture. TETS was subsequently investigated both as a rodenticide and as a pesticide [for an extensive review see Lauková et al. (2020)]. However, since human intoxications resulting in death; seizures; and, if victims survived exposure, neurologic deficits and spontaneous recurrent seizures have repeatedly occurred, the World Health Organization therefore banned the use of TETS (Lauková et al., 2020). But, because of its ease of manufacture and its impressive effectiveness in killing rats and mice, TETS continues to be used as a rodenticide in China and presents a real exposure

ABBREVIATIONS: AUC, area under the curve; EBOB, 1-(4-ethynylphenyl)-4-n-propyl-2,6,7-trioxabicyclo[2.2.2]octane; EC ${ }_{90}$, 90\% effective concentration; ECD, extracellular domain; EM, electron microscopy; GABA $A_{A}$ GABA receptor type A; NCA, noncompetitive antagonist; PCR, polymerase chain reaction; pdb, Protein Data Bank; RDL, resistant to dieldrin; REU, Rosetta energy unit; TBPS, tert-butylbicyclophosphorothionate; TETS, tetramethylenedisulfotetramine; TMD, transmembrane domain. 
risk to humans and a viable threat agent for inciting a masscasualty incident. Pharmacological experiments performed in the late 1950s demonstrating that sublethal doses of TETS could reverse pentobarbital-induced decreases in blood pressure and respiration in dogs (Haskell and Voss, 1957) suggested that TETS might be targeting $\mathrm{GABA}_{\mathrm{A}}$ receptors. This was later confirmed in binding assays. TETS, which is far more toxic to mice than to insects (Esser et al., 1991), has affinity for both mammalian $\mathrm{GABA}_{\mathrm{A}}$ receptors and the insect RDL receptor. Although the insect $R D L$ receptor is a homopentamer consisting of five identical subunits, mammalian $\mathrm{GABA}_{\mathrm{A}}$ receptors are typically composed of two $\alpha$, two $\beta$, and one $\gamma$ or $\delta$ subunit assembled as a pentamer around a central chloride-conducting pore (Olsen and Sieghart, 2008). In binding assays with rat brain synaptosomes, TETS displaces $\mathrm{GABA}_{\mathrm{A}}$ receptor blockers like $\left[{ }^{3} \mathrm{H}\right]$-1-(4-ethynylphenyl)4-n-propyl-2,6,7-trioxabicyclo[2.2.2] octane (EBOB) and $\left[{ }^{35} \mathrm{~S}\right]$ TBPS (Esser et al., 1991; Zhao et al., 2014), most likely by binding to the noncompetitive antagonist (NCA) site in the $\mathrm{GABA}_{\mathrm{A}}$ receptor pore. However, it was not known whether TETS displayed any subtype selectivity and where exactly it was binding. To address the first question, our group recently tested the potency of TETS on the major synaptic and extrasynaptic $\mathrm{GABA}_{\mathrm{A}}$ receptors associated with convulsant activity using whole-cell patch clamp and reported that TETS is most active on $\alpha_{2} \beta_{3} \gamma_{2 L}$ and $\alpha_{6} \beta_{3} \gamma_{2 L}$ GABA receptors (Pressly et al., 2018). Based on the observation that $\alpha_{2} \beta_{3} \gamma_{2}$ receptors make up $15 \%-20 \%$ of the GABA $_{\mathrm{A}}$ receptors in the mammalian central nervous system, we suggested that this receptor combination probably constitutes the most important $\mathrm{GABA}_{\mathrm{A}}$ receptor target for the seizure-inducing activity of TETS (Pressly et al., 2018). The results still left the question of the binding site unanswered.

The $\mathrm{GABA}_{\mathrm{A}}$ field has recently advanced substantially through the publication of numerous new structures (García-Nafría and Tate, 2020). As of now, $19 \mathrm{GABA}_{\mathrm{A}}$ receptor structures have been resolved in the closed, open, or desensitized state. Although the first structure was a homopentamer (Miller and Aricescu, 2014), more physiologically relevant heteropentamers containing $\alpha, \beta$, and $\gamma$ subunits have been elucidated using cryo-electron microscopy (EM) and have started to provide the field molecular insights into both ligand gating and the actions of clinically widely used drugs, such as the benzodiazepines (Masiulis et al., 2019; García-Nafría and Tate, 2020). Using three recently published $\mathrm{GABA}_{\mathrm{A}}$ receptor structures as templates, we here employed the Rosetta molecular modeling suite to build three homology models of the $\alpha_{2} \beta_{3} \gamma_{2}$ receptor, the most TETSsensitive $\mathrm{GABA}_{\mathrm{A}}$ receptor. Three different molecular modeling programs, RosettaLigand, Glide, and Swissdock, identified two possible TETS binding sites in the channel pore. Using a combination of site-directed mutagenesis and electrophysiology, we demonstrate that the relatively polar TETS molecule is interacting with all five subunits at the 6 '-position threonine ring of the pore-lining M2 segment at a site that is overlapping but not identical with the picrotoxinin binding site.

\section{Methods}

Study Design and Sample Sizes. This is an exploratory study for which we started with the working hypothesis that TETS is binding in the pore of the $\alpha_{2} \beta_{3} \gamma_{2 \mathrm{~L}} \mathrm{GABA}_{\mathrm{A}}$ receptor based on the fact that it behaves like a noncompetitive antagonist in electrophysiological experiments (Pressly et al., 2018) and displaces known pore blockers in radioligand binding assays (Esser et al., 1991; Zhao et al., 2014). Based on our previous experience with Rosetta modeling (Nguyen et al., 2017), we planned on generating 10,000 models for obtaining full-atom homology models of the $\alpha_{2} \beta_{3} \gamma_{2} \mathrm{GABA}_{\mathrm{A}}$ receptor and 50,000 docking trajectories for each ligand before identifying the 50 lowest-energy structures (see below). For the electrophysiological experiments, we also relied on our previous experience in mapping binding sites (Wulff et al., 2001; Zimin et al., 2010; Jenkins et al., 2011; Nguyen et al., 2017) and typically used five to eight independent recordings per data point when screening mutants or obtaining data for concentration-response curves.

Rosetta Molecular Modeling. We generated three fullatom homology models of the $\alpha_{2} \beta_{3} \gamma_{2}$ GABA $_{\mathrm{A}}$ receptor using the Rosetta molecular modeling suite (Rohl et al., 2004) with membrane environment-specific energy functions (YarovYarovoy et al., 2012). A model of the open state was based upon the X-ray structure of the $\beta_{3}$-extracellular domain (ECD)- $\alpha_{5}$-transmembrane domain (TMD) chimera, which had been reported in the presence and absence of the neurosteroid pregnanolone (Miller et al., 2017). The pregnanolonebound structure was chosen as a template [Protein Data Bank (pdb): 508F]. Two additional models were generated based on the cryo-EM structure of $\alpha_{1} \beta_{3} \gamma_{2}$ (Masiulis et al., 2019) with alprazolam (pdb: 6HUO) or with picrotoxinin bound (pdb: 6 HUG). Before homology modeling, all ligands and nanobodies were removed from the templates, and Jalview 2 (http://www.jalview.org/) was used to align the sequences of $\alpha_{2}, \beta_{3}$, and $\gamma_{2}$ with the templates. For the first model based on the $\beta_{3}$ ECD- $\alpha_{5}$ TMD chimera, the ECDs of $\alpha_{2}, \beta_{3}$, and $\gamma_{2}$ were aligned with the $\beta_{3} \mathrm{ECD}$, and the TMDs were aligned with the $\alpha_{5}$ TMD. The sequence homology between $\alpha_{2}$ and $\alpha_{5}$ or $\alpha_{1}$ is $73 \%$ and $70 \%$ in the TMD, respectively. The sequence homology between $\beta_{3}$ and $\alpha_{2}$ or $\gamma_{2}$ is $33 \%$ in the ECD. No additional loop modeling was performed since we were primarily interested in the well resolved TMD domains. All three homology models were refined using RosettaES (Frenz et al., 2017). Ten thousand models were generated, and the top-10 converging, lowest-energy models were selected and subjected to a final round of side-chain relaxation to minimize the energy. Before transferring the models to RosettaLigand, Glide, or Swissdock for ligand docking, posttranslational modified amino acids (e.g., glycosylated or palmitoylated) were converted to standard amino acids to avoid problems with minimization procedures in these programs. Verification of correct residues was performed visually. As described below, we then first probed the TMD with two ligands (picrotoxinin and EBOB) known to bind to the NCA site to verify how suitable our models were for proceeding to dock TETS.

RosettaLigand Docking of NCAs. Docking of picrotoxinin, EBOB, and TETS was performed with the RosettaLigand application (Meiler and Baker, 2006; Davis and Baker, 2009), which is comprised of three stages that progress from lowresolution conformational sampling and scoring to full-atom optimization. The RosettaLigand application with the Talaris2014 energy function was used for all docking procedures. In the first, low-resolution stage, the ligand is placed randomly within the binding site, and its center of mass is constrained to move within a $7-\AA$-diameter sphere. EBOB and picrotoxinin were placed according to their published binding sites (Chen et al., 2006; Masiulis et al., 2019) with their center of mass at 
the $6^{\prime}$ ring in the pore of the $\mathrm{GABA}_{\mathrm{A}}$ receptor. For TETS, we made the initial placements at six sites from position $0^{\prime}$ to position $20^{\prime}$ of the pore. Conformers were generated using OEChem, version 1.7.4 (OpenEye Scientific Software, Inc., Santa Fe, NM; www.eyesopen.com) and were then randomly rotated as a rigid body and scored for shape compatibility with the target protein (Hawkins et al., 2010; Hawkins and Nicholls, 2012). Please note that because of its caged structure, TETS only has one conformer. The best-scoring models were filtered by root-mean-square deviation to eliminate near duplicates, and one of the remaining models was selected at random to continue to the next stage. In the second, high-resolution stage, the Monte Carlo minimization protocol was employed, and ligand position and orientation were randomly perturbed by small $0.1-\AA$ and $3^{\circ}$ deviations: receptor side chains were repacked using a rotamer library; the ligand position, orientation, and torsions and protein side-chain torsions were simultaneously optimized using quasi-Newton minimization; and the end result was accepted or rejected based on the Metropolis criterion. Scoring used the full-atom Rosetta energy function with softened van der Waals repulsion. The side-chain rotamers were searched simultaneously during full repack cycles and one at a time in the rotamer trials cycles. The full repack makes $\sim 10^{6}$ random rotamer substitutions at random positions and accepts or rejects each based on the Metropolis criterion. Rotamer trials choose the single best rotamer at a random position in the context of the current state of the rest of the system with the positions visited once each in random order. The ligand is treated as a single residue, and its input conformers serve as rotamers during this stage. During the energy minimization step, the finely sampled rotamer library and soft-repulsive energy function allow access to off-rotamer conformations. In the third and final stage, a more stringent gradient-based minimization of the ligand position, orientation, and torsions as well as receptor torsions for both side chains and backbone were used. Scoring applies the same Rosetta energy function but with a hard-repulsive van der Waals potential, which creates a more rugged energy landscape that is better at discriminating native from non-native binding modes. Fifty thousand docking trajectories were generated for each channelligand pair, and the top 50 structures were selected according to the interface scores between the ligand and the protein. When a ligand converged on a common pose in a low-energy state with reoccurring interactions, we considered it converged. Rosetta energies comparing the three channels states on the arbitrary scale employed for the Rosette energy units (REUs) were calculated with the updated RosettaLigand application using the REF2015 energy function.

All molecular graphics were rendered using the UCSF Chimera software (Resource for Biocomputing, Visualization, and Informatics, San Francisco, CA) (Pettersen et al., 2004). Protein Data Bank format files of the closed/resting state of the $\alpha_{2} \beta_{3} \gamma_{2}$ receptor with and without TETS are provided in the Supplemental Data; pdb files of all other models with ligands docked are available upon request.

Glide. TETS, EBOB, and picrotoxinin were prepared for docking using the LigPrep function in Glide (Schrödinger, LLC, New York, NY, 2018) to generate rotamers, create 3D structures, and assign correct conformations. Ligands were docked into the RosettaES refined open-state model with the XP protocol using a 7-Å box at the same six sites in the pore from $0^{\prime}$ to $20^{\prime}$ as explored above with RosettaLigand. To identify possible ligand binding sites, we used the Glide default score of 0 as a cutoff and only accepted sites with negative scores.

Swissdock. The open-state homology model based on the structure of the $\beta_{3} \mathrm{ECD}-\alpha_{5} \mathrm{TMD}$ chimera (pdb: $5 \mathrm{O} 8 \mathrm{~F}$ ) was loaded into Swissdock (http://www.swissdock.ch) and probed blindly by docking TETS using $5000-15,000$ generated iterations. Swissdock uses the Chemistry at Harvard Macromolecular Mechanics force field (CHARMM) for estimating the chemical interactions and treats the protein as a grid. The accurate docking function was used with no flexibility allowed. The top results were examined visually, and only docking poses found in the TMD were further considered and are shown in Fig. 2.

Chemicals. Picrotoxinin and GABA were purchased from MilliporeSigma (St. Louis, MO). Diazepam was purchased from Tocris Bioscience (Bristol, UK). TETS was synthesized at the University of California, Davis as previously described (Zhao et al., 2014). Ten millimolar stocks of GABA were made fresh daily using Ringer's solution (see below for composition). Ten millimolar stocks of picrotoxinin and TETS were prepared in DMSO and diluted down into Ringer's solution only immediately before application onto the cell to avoid any hydrolysis of picrotoxinin (Pressly et al., 2020). The identity of TETS and picrotoxinin was confirmed by ${ }^{1} \mathrm{H}$ - and ${ }^{13} \mathrm{C}-\mathrm{NMR}$; purity of TETS was tested by GC/mass spectrometry or highpressure liquid chromatography/mass spectrometry and found to be $>98 \%$ based on total ion fragment analysis. Both TETS and picrotoxinin waste were treated with nitric acid and disposed of using the waste accumulation program at University of California, Davis. The synthesis and chemical characterization of the TETS haptens used in Fig. 8 was previously described (Barnych et al., 2017). Hapten 2a is ( \pm )-( $1 R, 3 S, 8 R)$-2,7-dithia1,3,6,8-tetraazatricyclo[4.3.1.1 $\left.1^{3,8}\right]$ undecane $2,2,7,7$-tetraoxide); Hapten $4 \mathrm{a}$ is $(( \pm)-(1 R, 5 R)-3,7$-dimethyl-2,6-dithia- $1,3,5,7-$ tetraazabicyclo[3.3.1]nonane 2,2,6,6-tetraoxide); Hapten $6 \mathrm{~b}$ is $((1 R, 3 S, 5 r, 7 r)$-7-amino-2-thia-1,3,5-triazaadamantane 2,2 dioxide).

Cell Culture. The human GABA $A_{\mathrm{A}}$ receptors $\alpha_{2}, \alpha_{6}, \beta_{3}$, and $\gamma_{2 \mathrm{~L}}$ cloned into pcDNA3.1 expression vectors were a generous gift from Dr. Robert L. Macdonald, Vanderbilt University, Nashville, TN. L929 cells, a mouse fibroblast cell line (CCL-1), were obtained from American Type Culture Collection (Manassas, VA). L929 cells were cultured in Dulbecco's modified Eagle's medium (Lonza, Basel, Switzerland) supplemented with $10 \% \mathrm{FBS}, 100 \mathrm{U} / \mathrm{ml}$ penicillin, and $100 \mathrm{mg} / \mathrm{ml}$ streptomycin (Invitrogen; ThermoFisher, Grand Island, NY) and maintained in humidified $95 \%$ air and $5 \% \mathrm{CO}_{2}$ at $37^{\circ} \mathrm{C}$. L929 cells were transfected using FuGENE 6 (ThermoFisher) transfection reagent in Opti-MEM reduced serum medium (Life Technologies, Benicia, CA) with an equal amount of each of the subunits (1:1:1) in combination with GFP expressed from the pEGFP-C1 vector (Invitrogen). The ratio of total cDNA to transfection reagent was $2: 1$. Cells were detached by trypsinization 48 hours post-transfection, washed, and plated onto poly-L-lysine-coated glass coverslips. Transfected cells were identified as GFP-expressing cells using an epifluorescence microscope for electrophysiological whole-cell voltageclamp studies. Correct incorporation of the $\gamma$ subunit was tested by determining sensitivity to diazepam, a $\mathrm{GABA}_{\mathrm{A}}$ receptor positive allosteric modulator that binds at the $\alpha / \gamma$ interface as previously described (Pressly et al., 2018). 
Mutagenesis. Mutagenesis primers were designed with PrimerX software (http://www.bioinformatics.org/primerx) and were $20-30$ base pairs in length with a five to eight base pair overhang on the $3^{\prime}$ end. Appropriate melting temperatures were calculated with NEB Tm Calculator (https:// tmcalculator.neb.com/main). The primers were synthesized by ThermoFisher and were diluted to $10 \mu \mathrm{M}$ from $100 \mu \mathrm{M}$ stocks for polymerase chain reaction (PCR). NEB Phusion High-Fidelity DNA polymerase (ThermoFisher) was used to amplify the template DNA (10-100 ng). PCR cycling conditions were as follows: initial denaturation at $98^{\circ} \mathrm{C}$ for 30 seconds; 35 cycles of denaturation at $98^{\circ} \mathrm{C}$ for 10 seconds, annealing at $48-68^{\circ} \mathrm{C}$ for 30 seconds, and extension at $72^{\circ} \mathrm{C}$ for 150 seconds; and final extension at $72^{\circ} \mathrm{C}$ for 10 minutes. One percent DMSO was added to the PCR reactions. After the PCR, the DNA was digested with DpnI (New England Biolabs, Ipswich, MA) overnight at $37^{\circ} \mathrm{C}$. Purity of product was assessed on $1 \%$ agarose gels. Transformations were performed with 1-5 $\mu$ l of PCR product for each mutant and $100 \mu \mathrm{l}$ MAX Efficiency DH10B competent Escherichia coli using heat shock according to the manufacturer's protocol. Successfully transformed cells gave visible colonies on either ampicillin or kanamycin selection plates. Mutant sequences were confirmed via sequencing using ABI 3730 Capillary Electrophoresis Genetic Analyzers (University of California, Davis DNA Sequencing Facility). Mutants were deemed functional if they produced at least $200 \mathrm{pA}$ of current in response to $100 \mu \mathrm{M}$ GABA and were sensitive to positive modulation by diazepam. The following mutants did not produce functional currents in our hands: $\alpha_{2} \mathrm{~T} 6^{\prime} \mathrm{F}, \alpha_{2} \mathrm{~V} 2^{\prime} \mathrm{A}, \beta_{3} \mathrm{~T} 6^{\prime} \mathrm{F}, \beta_{3} \mathrm{~T} 6^{\prime} \mathrm{W}$, and $\gamma_{2} \mathrm{~T} 6^{\prime} \mathrm{Y}$.

Electrophysiological Recordings. Whole-cell voltageclamp recordings were performed at room temperature with an EPC-10 HEKA amplifier (HEKA Elektronik, Lambrecht, Germany). Cells were bathed in an external Ringer's solution consisting of $160 \mathrm{mM} \mathrm{NaCl}, 4.5 \mathrm{mM} \mathrm{KCl}, 1 \mathrm{mM} \mathrm{MgCl}_{2}, 2 \mathrm{mM}$ $\mathrm{CaCl}_{2}$, and $10 \mathrm{mM}$ HEPES, $\mathrm{pH} 7.4$ and $308 \mathrm{mOsm}$. Recording electrodes were pulled from soda lime glass microhematocrit tubes (Kimble Chase, Rochester, NY) and fire-polished to resistances of 1.8-3 $\mathrm{M} \Omega$. Electrodes were filled with an internal solution consisting of $154 \mathrm{mM} \mathrm{KCl}, 2 \mathrm{mM} \mathrm{CaCl}_{2}$, $1 \mathrm{mM} \mathrm{MgCl}, 10 \mathrm{mM}$ HEPES, and $10 \mathrm{mM}$ EGTA, $\mathrm{pH} 7.2$ and $302 \mathrm{mOsm}$. Cells were voltage-clamped at $-80 \mathrm{mV}$, and control currents were recorded under the local application of $\mathrm{EC}_{90} \mathrm{GABA}(100 \mu \mathrm{M})$ for 5 seconds to the patch-clamped cell using an 8-channel pinch valve-controlled gravity perfusion system (VC3-8xG system; ALA Scientific) positioned within $100 \mu \mathrm{m}$ of the cell. GABA applications were followed by a 50-second wash with Ringer's solution. Washes and TETS additions to the chamber were performed through a separate, syringe-driven perfusion system with a short perfusion line (length $236 \mathrm{~mm}$, line volume $210 \mu \mathrm{l}$ ) and with a volume $(2 \mathrm{ml})$ that exchanged the chamber volume five times. TETS was allowed to sit for 3 minutes on the cell before reapplication of $\mathrm{EC}_{90}$ GABA directly onto the cell through the gravity perfusion system. Percentage of block was calculated using the area under the curve (AUC). One cell was used per concentration of the convulsant. Cells that became leaky during the experiment or that did not produce the same magnitude of response to $\mathrm{EC}_{90}$ GABA twice before the experiment and after washout of TETS were excluded from the analysis. For screening of mutant channels, $50 \mu \mathrm{M}$ TETS and $\mathrm{EC}_{90}$ GABA were used to evaluate whether the mutation affected TETS potency. Percentage of current blocked (mean \pm S.D. from $n=5-8$ cells per mutant) was analyzed with one-way ANOVA followed by Dunnett's test to compare the means with the wild-type control and to correct for multiple comparisons. ${ }^{*} P<0.05$; $* * P<0.01$; and $* * * P<0.001$.

Concentration-response curves for selected mutants were constructed by testing varying concentrations of TETS or picrotoxinin for their ability to block currents elicited by $100 \mu \mathrm{M}$ GABA (=EC 90 GABA). For analysis of current blockade, the area under the current curve $\left(\mathrm{AUC}_{\mathrm{Max}}\right)$ was determined for the control $\left(\mathrm{EC}_{90} \mathrm{GABA}\right)$ and the $\mathrm{AUC}_{\mathrm{Ex}}$ after exposure. $\left[\mathrm{AUC}_{\mathrm{Ex}}\right] /\left[\mathrm{AUC}_{\mathrm{Max}}\right] \times 100=\%$ Current Blocked. Data analysis and data fitting to the Hill equation to obtain $\mathrm{EC}_{50}$ or $\mathrm{IC}_{50}$ values was performed using Origin 9.1 (OriginLab Corporation, Northampton, MA) software. Individual data points are presented as mean \pm S.D. from five to eight independent recordings. $\mathrm{EC}_{50}$ and $\mathrm{IC}_{50}$ values are presented with $95 \%$ confidence intervals. Concentration-response curves were compared using an extra sum-of-squares F test (GraphPad Prism8; GraphPad Software, La Jolla, CA).

\section{Results}

Generation and Validation of a Homology Model of the $\boldsymbol{\alpha}_{\mathbf{2}} \boldsymbol{\beta}_{\mathbf{3}} \gamma_{\mathbf{2}} \mathbf{G A B A}_{\mathbf{A}}$ Receptor. Since the $\alpha_{2} \beta_{3} \gamma_{2 \mathrm{~L}} \mathrm{GABA}_{\mathrm{A}}$ receptor constitutes the most sensitive and important target for the proconvulsant activity of TETS (Pressly et al., 2018), we decided to delineate the TETS binding site in this particular subunit combination. For experimental work and molecular modeling, it should be noted that TETS is neither acidic nor basic and therefore uncharged. It also only has a single conformer. Based on our previously published findings, TETS is a noncompetitive $\mathrm{GABA}_{\mathrm{A}}$ receptor antagonist, which, similar to picrotoxinin, cannot be competed off by raising GABA concentrations (Pressly et al., 2018). As such, TETS could potentially be binding to an open, a postactivation, or a closed state of the $\alpha_{2} \beta_{3} \gamma_{2 L}$ receptor.

We had previously observed that TETS is more potent when $\mathrm{GABA}_{\mathrm{A}}$ receptors are preincubated with TETS (Pressly et al., 2018) than when TETS and GABA are applied together, suggesting that TETS could be interacting with a closed state of the channel. This experimental setup is illustrated in Fig. $1 \mathrm{~A} ; \alpha_{2} \beta_{3} \gamma_{2 \mathrm{~L}}$ receptors were activated by a 5 -second application of $100 \mu \mathrm{M}$ GABA directly to the patch-clamped cell. GABA is then washed away, and TETS is perfused into the recording chamber and allowed to equilibrate for 3 minutes. When GABA is then reapplied to the cell, it induces a much smaller current with virtually no further enhancement of current decay, suggesting that TETS can block closed channels. A 50-second wash of the chamber with Ringer's solution completely reversed the TETS effect (Fig. 1A). In contrast, if TETS and GABA were perfused together directly onto the cell with no preincubation, the current induced by $100 \mu \mathrm{M}$ GABA was larger and displayed an accelerated rate of current decay (Fig. 1B), suggesting open-channel block or enhanced desensitization. TETS thus resembles picrotoxinin, which is often described as an openchannel blocker with some additional "allosteric effects" (Xu et al., 1995; Othman et al., 2012; Olsen et al., 2019). Based 

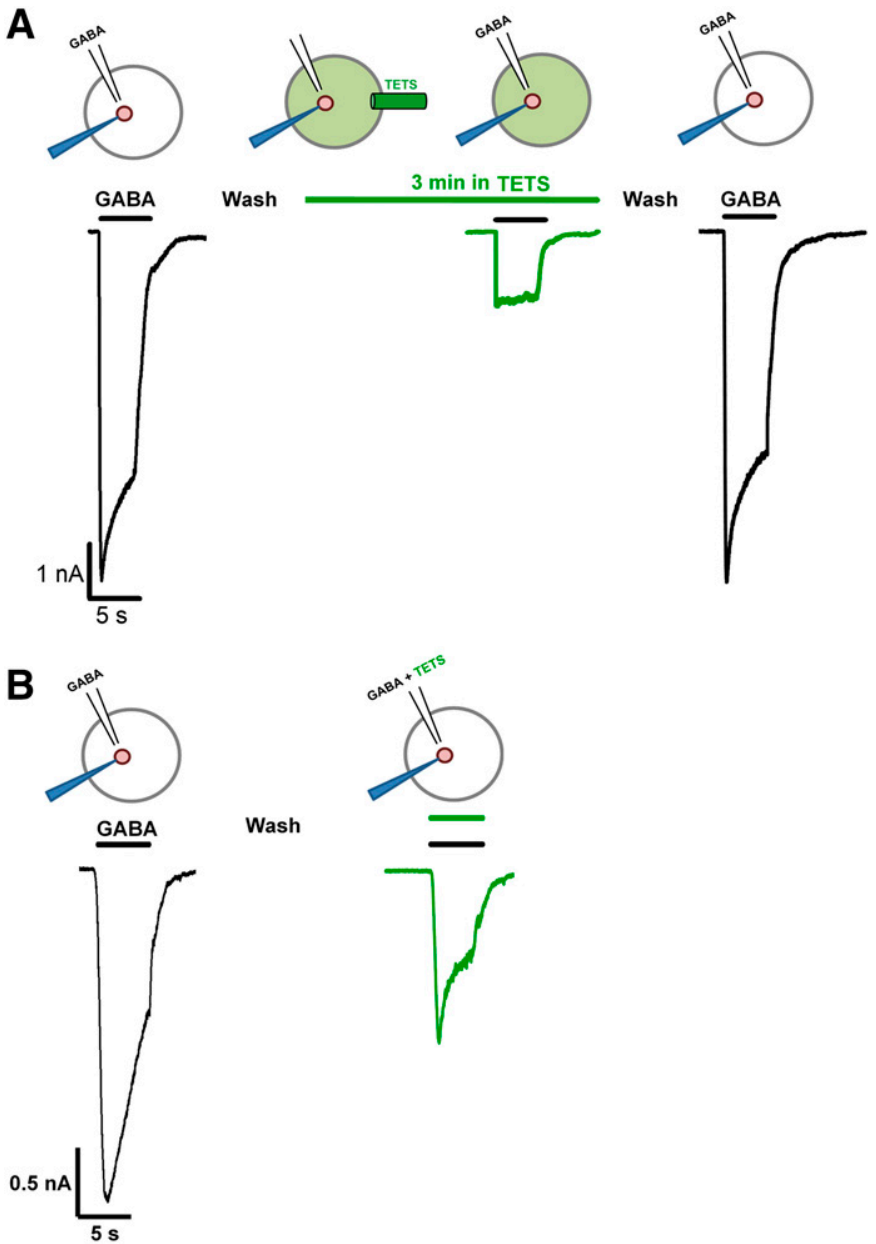

Fig. 1. TETS can block closed and open $\alpha_{2} \beta_{3} \gamma_{2 \mathrm{~L}}$ receptors. (A) Chloride currents were activated by a 5 -second application of $100 \mu \mathrm{M}$ GABA directly to the patch-clamped cell. GABA was then washed out by a 50-second wash of the chamber with Ringer's solution. One minute later, $50 \mu \mathrm{M}$ of TETS was perfused into the chamber and allowed to equilibrate for 3 minutes. GABA $(100 \mu \mathrm{M})$ was then reapplied directly to the cell with TETS in the bath. A subsequent 50-second wash of the chamber with Ringer's solution completely reversed the TETS effect. (B) After a control current was elicited by direct application of $100 \mu \mathrm{M}$ GABA, TETS and GABA were perfused together directly onto the cell with no preincubation.

on this preliminary "look" at the mechanism of TETS blockade, we postulated that TETS is a pore blocker with fast on and off rates that is probably capable of blocking multiple states of $\mathrm{GABA}_{\mathrm{A}}$ receptors.

At the time, when we started this work in 2017, the most suitable template that was available for homology modeling was the X-ray structure of $\beta_{3} \mathrm{ECD}-\alpha_{5} \mathrm{TMD}$ chimera (Fig. $2 \mathrm{~A}$ ), which had been reported in the presence and absence of the neurosteroid pregnanolone (Miller et al., 2017). Before homology modeling, pregnanolone and the nanobodies, which had served as crystallization aids, were removed from the $3.2-\AA$ resolution structure (Fig. $2 \mathrm{~A}$ ). We then aligned the sequences of the ECDs of $\alpha_{2}, \beta_{3}$, and $\gamma_{2}$ with the $\beta_{3}$ ECD and the TMD sequences of these subunits with the $\alpha_{5}$ TMD part of the chimeric structure and generated a full-atom homology model of the $\alpha_{2} \beta_{3} \gamma_{2} \mathrm{GABA}_{\mathrm{A}}$ receptor using the Rosetta molecular modeling suite (Rohl et al., 2004) followed by RosettaES refinement (Frenz et al., 2017). In the resulting homology model of the $\alpha_{2} \beta_{3} \gamma_{2}$ GABA $_{\mathrm{A}}$ receptor shown in Fig. 2B, the two $\alpha_{2}$ subunits are colored in blue, the two $\beta_{3}$ subunits are colored in red, and $\gamma_{2}$ is shown in yellow. The pore is formed by five M2 helices and is $4.26 \AA$-wide at the desensitization gate on the intracellular side ( -2 ' proline) similar to its template, which is $4.3 \AA$-wide at this position and therefore presumably open. Based on the high degree of homology in M2 between the different $\mathrm{GABA}_{\mathrm{A}}$ subunits (Fig. 2C), the Arg residue at the cytoplasmic end of M2 is typically designated $0^{\prime}$, and the residue at the extracellular end of the pore is designated $20^{\prime}$. Residues below the $0^{\prime} \mathrm{Arg}$ are counted with negative numbers (Fig. 2D). This commonly used residue numbering system makes it easy to compare mutagenesis and modeling work across different $\mathrm{GABA}_{\mathrm{A}}$ receptor subtypes, and we are therefore also using it here.

To validate our $\alpha_{2} \beta_{3} \gamma_{2}$ homology model, we first docked two widely used $\mathrm{GABA}_{\mathrm{A}}$ antagonists: picrotoxinin and EBOB. Both compounds are known to bind to the NCA site in the pore of the $\mathrm{GABA}_{\mathrm{A}}$ receptor (Chen et al., 2006; Olsen, 2006). The most detailed work on the NCA site has been performed with the sesquiterpene picrotoxinin, which has been suggested to interact with residues in the $2^{\prime}$ and $6^{\prime}$ positions based on mutagenesis in the $\alpha_{1} \beta_{1} \gamma_{2}$ and the $\alpha_{1} \beta_{2} \gamma_{2}$ $\mathrm{GABA}_{\mathrm{A}}$ receptors (Xu et al., 1995; Erkkila et al., 2008; Ng et al., 2016). Other compounds binding to the NCA site have been explored using the insect RDL channel and the probably unphysiological $\beta_{3}$ homopentamer in $\left[{ }^{3} \mathrm{H}\right] \mathrm{EBOB}$ or $\left[{ }^{35} \mathrm{~S}\right]$ TBPS binding assays (Esser et al., 1991; Chen et al., 2006). Both TETS and picrotoxinin displace the larger EBOB, suggesting that all three compounds interact with overlapping sites. Based on mutagenesis experiments, EBOB has been suggested to "sit upright" in the pore, blocking the channel's ability to conduct chloride by stretching from residue $2^{\prime}$ in the lower pore to residue $9^{\prime}$ in the open homopentameric $\beta_{3}$ $\mathrm{GABA}_{\mathrm{A}}$ receptors (Chen et al., 2006).

Using the RosettaLigand method, we docked EBOB and picrotoxinin into the pore of our open $\alpha_{2} \beta_{3} \gamma_{2}$ homology model (Supplemental Fig. 1) at the NCA site in the M2 segment, generated 50,000 docking trajectories, and collected the top 50 models with the energetically most-favorable interface scores between the ligand and the protein. For EBOB, Rosetta converged on a dominant binding pose where it is seen stretching from the $2^{\prime}$ to the $9^{\prime}$ ring in keeping with the previously mapped binding site (Chen et al., 2006). One ether oxygen in EBOB's trioxabicyclooctane cage is hydrogen bonding to $\beta_{3} \mathrm{~T} 6^{\prime}$, whereas $\alpha_{2} \mathrm{~V} 2^{\prime}$, the methyl group of $\alpha_{2} \mathrm{~T} 6^{\prime}$, and $\beta_{3} \mathrm{L9}^{\prime}$ are making van der Walls interactions (Supplemental Fig. 1A). For picrotoxinin, Rosetta converged on a frequently sampled binding pose (Supplemental Fig. 1B) in which picrotoxinin forms one hydrogen bond with $\gamma_{2} \mathrm{~S} 2^{\prime}$ and two hydrogen bonds with $\beta_{3} \mathrm{~T} 6^{\prime}$ and $\gamma_{2} \mathrm{~T}^{\prime}$ at the so-called $\mathrm{T} 6^{\prime}$ ring or loop, where five threonine residues encircle the permeation pathway of the channel (Fig. 1C). This binding pose agrees well with previously performed mutagenesis showing that the $\mathrm{T}^{\prime}$ ' ring is crucial for picrotoxinin binding (Erkkila et al., 2008; Ng et al., 2016). The Rosetta model is also in good agreement with the subsequently published $\alpha_{1} \beta_{3} \gamma_{2}$ cryo-EM structure with picrotoxinin bound, where picrotoxinin is observed between the L9' ring and the $2^{\prime}$ ring with major interactions, presumably hydrogen bonds, to the T6' ring (Masiulis et al., 2019). 


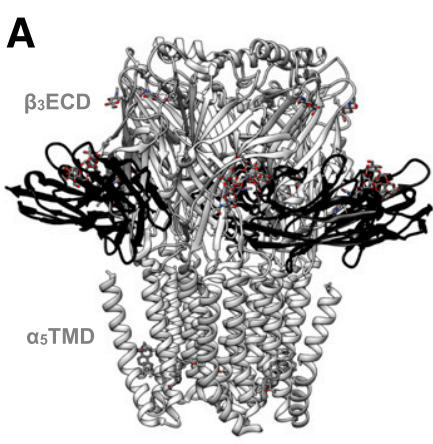

$\beta_{3} E C D-\alpha_{5} T M D X$-ray structure

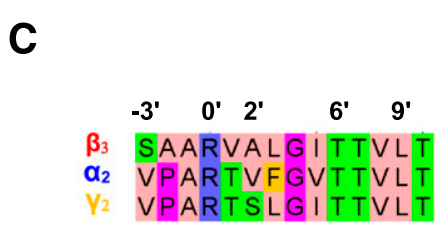

B

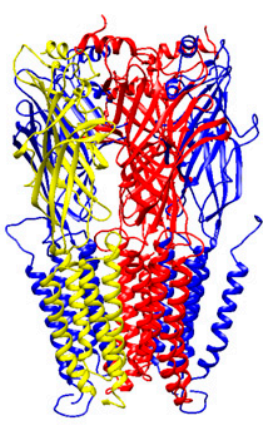

$\alpha_{2} \beta_{3} \gamma_{2}$ Homology model

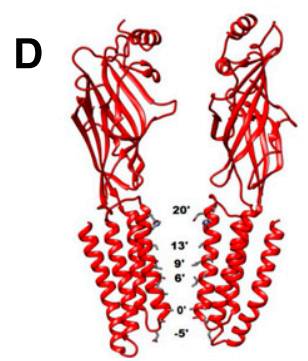

Fig. 2. Generation of a homology model of the $\alpha_{2} \beta_{3} \gamma_{2} \mathrm{GABA}_{\mathrm{A}}$ receptor. (A) The X-ray structure of the $\beta_{3}$ ECD- $\alpha_{5}$ TMD served as a template. (B) The Rosetta-generated homology model of the $\alpha_{2} \beta_{3} \gamma_{2}$ in the open state. The receptor is color-coded as follows: $\alpha_{2}$ (blue), $\beta_{3}$ (red), $\gamma_{2}$ (yellow). (C) Sequence alignment of $\alpha_{2}, \beta_{3}$, and $\gamma_{2}$ in the M2 segment. (D) Common numbering of pore-lining residues in $\mathrm{GABA}_{\mathrm{A}}$ receptors.

Searching for the TETS Binding Site in the $\alpha_{2} \beta_{3} \gamma_{2}$ GABA A $_{\mathbf{A}}$ Receptor. After finding that the RosettaLigand method converged on reasonable binding poses for picrotoxinin and EBOB at the NCA binding site in our $\alpha_{2} \beta_{3} \gamma_{2}$ homology model, we next used RosettaLigand to search for the putative TETS binding site in the lower pore. Since the $\mathrm{GABA}_{\mathrm{A}}$ receptor pore is long and narrow, TETS was randomly placed into 7-Å-diameter spheres at six sites between residue $0^{\prime}$ and $20^{\prime}$ of the pore. TETS was minimized using the three stages of the RosettaLigand application in all six positions, and convergence of the top 50 models was used to predict a putative binding site. Figure $3 \mathrm{~A}$ shows the six sites that were probed with Rosetta. TETS converged in two of these sites, which are highlighted in green as site-A (Fig. 3A) and site-C (Fig. 3A). To the right, close-up views of both sites are shown, which we used to guide further mutagenesis work. Site-A is at the $-2^{\prime}$ to $-3^{\prime}$ region of the M2 portion of our $\alpha_{2} \beta_{3} \gamma_{2}$ homology model. Here, as shown in a view from below, one oxygen in one of the sulfonamides of TETS is accepting two hydrogen bonds from the hydroxyl group and the $\mathrm{NH}$ of the amide backbone of $\beta_{3} \mathrm{~S}-3^{\prime}$, whereas the other sulfonamide oxygen is hydrogen bonding with the $\mathrm{NH}$ of the amide backbone of $\beta_{3} \mathrm{P}-2^{\prime}$. Site-C is at the $\mathrm{T} 6^{\prime}$ ring of our $\alpha_{2} \beta_{3} \gamma_{2}$ homology model. In 21 out of the top 50 energetically most-favorable binding poses, the hydroxy groups of the $\beta_{3} \mathrm{~T} 6^{\prime}$ and the $\gamma_{2} \mathrm{~T}^{\prime}$ hydrogen bond to the sulfonamide group of TETS, whereas one of the tertiary nitrogen atoms in the central cage of TETS accepts an additional hydrogen bond from the $\gamma_{2} \mathrm{~S} 2^{\prime}$ (Fig. 3A). In an alternative and also relatively frequently sampled binding pose ( 8 of 50), TETS hydrogen bonds with $\alpha_{2} \mathrm{~T} 6^{\prime}$ while maintaining the contact with $\gamma_{2}$ (unpublished data). The remainder of the energetically favorable poses show hydrogen

bonds with at least one $\mathrm{T} 6^{\prime}$ residue indicating the importance of this position.

To not solely rely on RosettaLigand, we also used Glide and Swissdock software to predict putative TETS binding sites in the Rosetta-generated homology model. With Glide, a program that, like RosettaLigand, requires the ligand to be randomly placed into predefined 7-Å boxes, we probed the M2 segment of the pore in our homology model using the same six starting positions as in Rosetta. Glide XP identified energetically favorable binding poses for TETS at site-A and site-C (shown in purple in Fig. 3B) but failed to identify possible binding poses in the other positions. At site-C in Glide, TETS is again positioned at the $\mathrm{T} 6^{\prime}$ ring, whereas at site-A, TETS sits further away from the pore than in RosettaLigand between the $\beta_{3}$ and $\alpha_{2}$ subunits but again forms a hydrogen bond with $\beta_{3} \mathrm{~S}-3^{\prime}$. With Swissdock, in contrast, we evaluated the entire $\alpha_{2} \beta_{3} \gamma_{2}$ homology model, including the extracellular domain and the outside of the transmembrane domains because of how Swissdock operates. Modeling jobs are submitted to a server, and the program offers users minimal control of the docking process. Swissdock identified two possible binding sites as shown in cyan in Fig. 3C: one site again in the pore at the $\mathrm{T} 6^{\prime}$ ring and a second site in the lipid-exposed region of the $\gamma_{2}$ subunit. We discarded the second site as unlikely because it was incompatible with the experimentally observed displacement of picrotoxinin and EBOB by TETS.

Since all three molecular modeling programs identified a putative binding site for TETS at the $\mathrm{T} 6^{\prime}$ ring, and two of the three programs suggested a potential second site at the lower entrance to the pore, we decided to probe both sites by mutagenesis directed by the binding poses suggested by RosettaLigand (Fig. 3A).

Mutations in the $\alpha_{2}$ and $\beta_{3}$ Subunits of the $\alpha_{2} \beta_{3} \gamma_{2}$ GABA $_{\mathbf{A}}$ Receptor Change TETS Potency in Electrophysiology. To determine whether mutations of any of the identified residues would change the ability of TETS to block chloride currents carried by $\alpha_{2} \beta_{3} \gamma_{2} \mathrm{GABA}_{\mathrm{A}}$ receptors, we transiently expressed wild-type and mutant receptors in L929 cells and studied them by whole-cell patch clamp. Correct incorporation of the $\gamma$ subunit was confirmed by testing diazepam sensitivity as previously described (Pressly et al., 2018). As an initial screen for identifying mutations that substantially affect TETS affinity, we tested TETS at a concentration of $50 \mu \mathrm{M}$, which is roughly 100 -fold above its $\mathrm{IC}_{50}$ of $480 \mathrm{nM}$ for blocking $\alpha_{2} \beta_{3} \gamma_{2} \mathrm{GABA}_{\mathrm{A}}$ receptors at the $\mathrm{GABA} \mathrm{EC}_{90}(100 \mu \mathrm{M})$ for this receptor subtype (Pressly et al., 2018).

Since our model suggested that TETS is interacting either with residues at the $-3^{\prime}$ and $-2^{\prime}$ positions (Fig. $3 \mathrm{~A}$, site-A) at the entrance to the permeation pathway (Fig. 2D) or, alternatively, with residues at the $2^{\prime}$ and $6^{\prime}$ rings (Fig. 3A Site-C), we started by introducing mutations into the $\beta_{3}$ and $\gamma_{2}$ subunits at the positions where RosettaLigand had suggested hydrogen-bonding contacts. In Fig. 4 we showcase cutaways of the transmembrane domains for each individual subunit, rendering the mutated pore-lining residues as stick models. (It should be kept in mind that mutations in the $\alpha$ or $\beta$ subunit always introduce changes in two of the five subunits of the heteropentameric channel consisting of two $\alpha$, two $\beta$, and one $\gamma$ subunit.) For the $\beta_{3}$ subunit, we studied a total of four mutants, $\mathrm{S}-3^{\prime} \mathrm{F}, \mathrm{A} 2^{\prime} \mathrm{S}, \mathrm{T} 6^{\prime} \mathrm{C}$, and L9'Y, thus "walking" through the length of the pore starting from the intracellular side (Fig. 4A). Of these four 


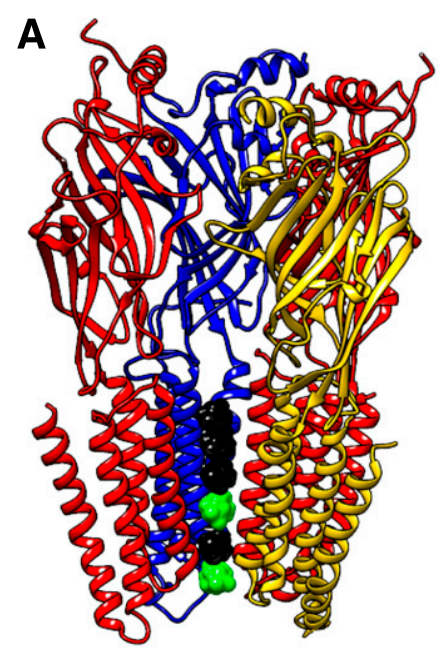

Rosetta

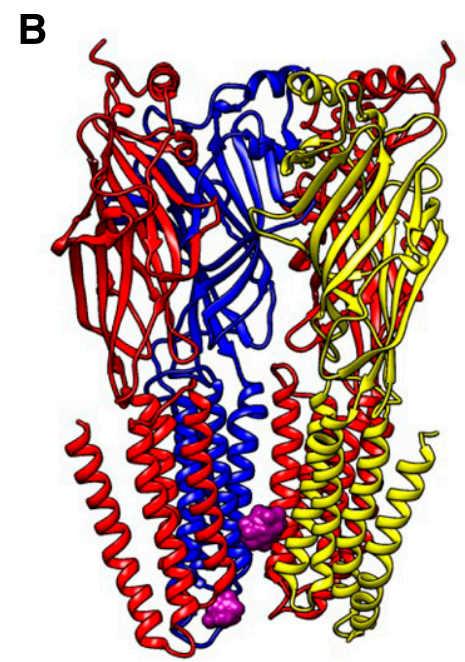

Glide XP
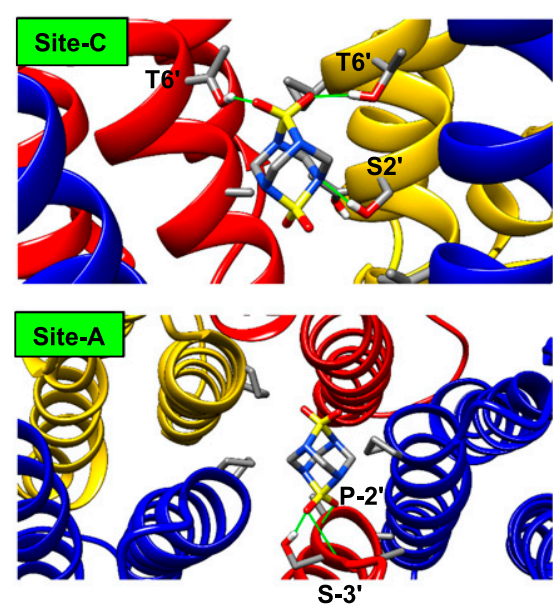

C

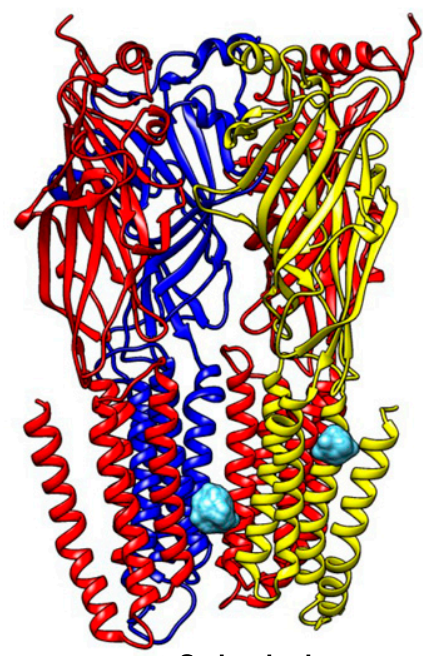

Fig. 3. Searching for the TETS binding site in the open-state model. The receptor is color-coded as follows: $\alpha_{2}$ (blue), $\beta_{3}$ (red), and $\gamma_{2}$ (yellow). (A) "Walk" through the pore of the $\alpha_{2} \beta_{3} \gamma_{2}$ homology model in six boxes of 7-Å diameter in RosettaLigand, which identified site-A and site-C as possible binding sites. (B) TETS binding sites suggested by Glide XP. (C) TETS binding sites suggested by Swissdock. mutants, $\mathrm{A} 2$ ' $\mathrm{S}$ and $\mathrm{T} 6{ }^{\prime} \mathrm{C}$ showed appreciable reductions in TETS activity, suggesting that $\mathrm{T}^{\prime}{ }^{\prime}$ and $\mathrm{A} 2^{\prime}$ in the $\beta_{3}$ subunit are important for TETS binding. In contrast, there was no change in TETS activity with the L9' Y mutation, suggesting that TETS is not extending as far up into the pore as picrotoxinin or EBOB. $\beta_{3}$ mutants with larger amino acid substitution in the $\mathrm{T} 6^{\prime}$ position, $\mathrm{T} 6^{\prime} \mathrm{F}$ and $\mathrm{T} 6^{\prime} \mathrm{W}$, did not express in our hands. For the $\gamma_{2}$ subunit, we studied a total of six mutants, P-2'H, P-2'F, S2'G, S2'F, S2'A, and T6'C (Fig. 4B). Of these six mutants, which were primarily designed to closely probe the -2 ' and $2^{\prime}$ site by sterically disturbing it, only the $\mathrm{T} 6{ }^{\prime} \mathrm{C}$ mutation reduced TETS activity, confirming the RosettaLigand prediction that TETS is probably hydrogen bonding with $\gamma_{2} \mathrm{~T} 6^{\prime}$ and $\beta_{3} \mathrm{~T} 6^{\prime}$. Our attempts to obtain a larger effect by mutating the $\mathrm{T} 6^{\prime}$ position in $\gamma_{2}$ failed because the $\gamma_{2} \mathrm{~T}^{\prime} \mathrm{Y}$ mutant was not functional.

Taken together, these results ruled out site-A as a possible TETS binding site, and we therefore next focused our attention on site-C by mutating the $2^{\prime}$ and $6^{\prime}$ residues in the $\alpha_{2}$ subunit as suggested by the alternative, less frequently sampled RosettaLigand poses for site-C. Of the four tested $\alpha_{2}$ mutants, $\mathrm{V} 2^{\prime} \mathrm{W}$, T6'M, T6'D, and T6'S (Fig. 4A), all three T6' mutants notably reduced TETS activity, whereas the mutation in the $2^{\prime}$ position had no impressive effect, demonstrating that $\mathrm{T}^{\prime}$ is a critical residue for TETS binding on the $\alpha_{2}$ subunit. The $\alpha_{2} \mathrm{~V} 2$ 'A and T6' $\mathrm{F}$ mutants did not express in our hands and could not be studied. Similar results were obtained with the $\alpha_{6} \beta_{3} \gamma_{2}$ receptor, which is blocked by TETS with an $\mathrm{IC}_{50}$ of $400 \mathrm{nM}$ (Pressly et al., 2018). Mutating $\beta_{3}$ in the $-3^{\prime}$ position $\left(\mathrm{S}-3^{\prime} \mathrm{F}\right)$ or $\gamma_{2}$ in the $2^{\prime}$ position $\left(\mathrm{S} 2^{\prime} \mathrm{G}\right)$ did not notably alter the blocking potency of $50 \mu \mathrm{M}$ TETS (unpublished data), whereas mutating V2' in $\alpha_{6}$ reduced the ability of $50 \mu \mathrm{M}$ TETS to block currents elicited by EC $_{90}$ GABA to $38 \%$ (S.D. $4.8 \%, n=5$ ).

A Resting/Inactivated State Model of the $\alpha_{2} \beta_{3} \gamma_{2}$ $\mathrm{GABA}_{\mathrm{A}}$ Receptor Produces a "Tighter," Energetically More-Favorable Model of the TETS Receptor Site. Although the mutagenesis experiments confirmed that TETS is interacting with site-C and not with site-A, we were somewhat concerned that TETS was so mobile in the pore during energy minimization in our open-state model, resulting in multiple possible binding poses instead of a single predominant pose. Specifically, TETS was seen to be sampling several positions at the $\mathrm{T}^{\prime}$ ' ring and was alternatively hydrogen bonding with either $\beta_{3} \mathrm{~T}^{\prime} 6$ or $\alpha_{2} \mathrm{~T}^{\prime} 6$ while maintaining contact with 
A
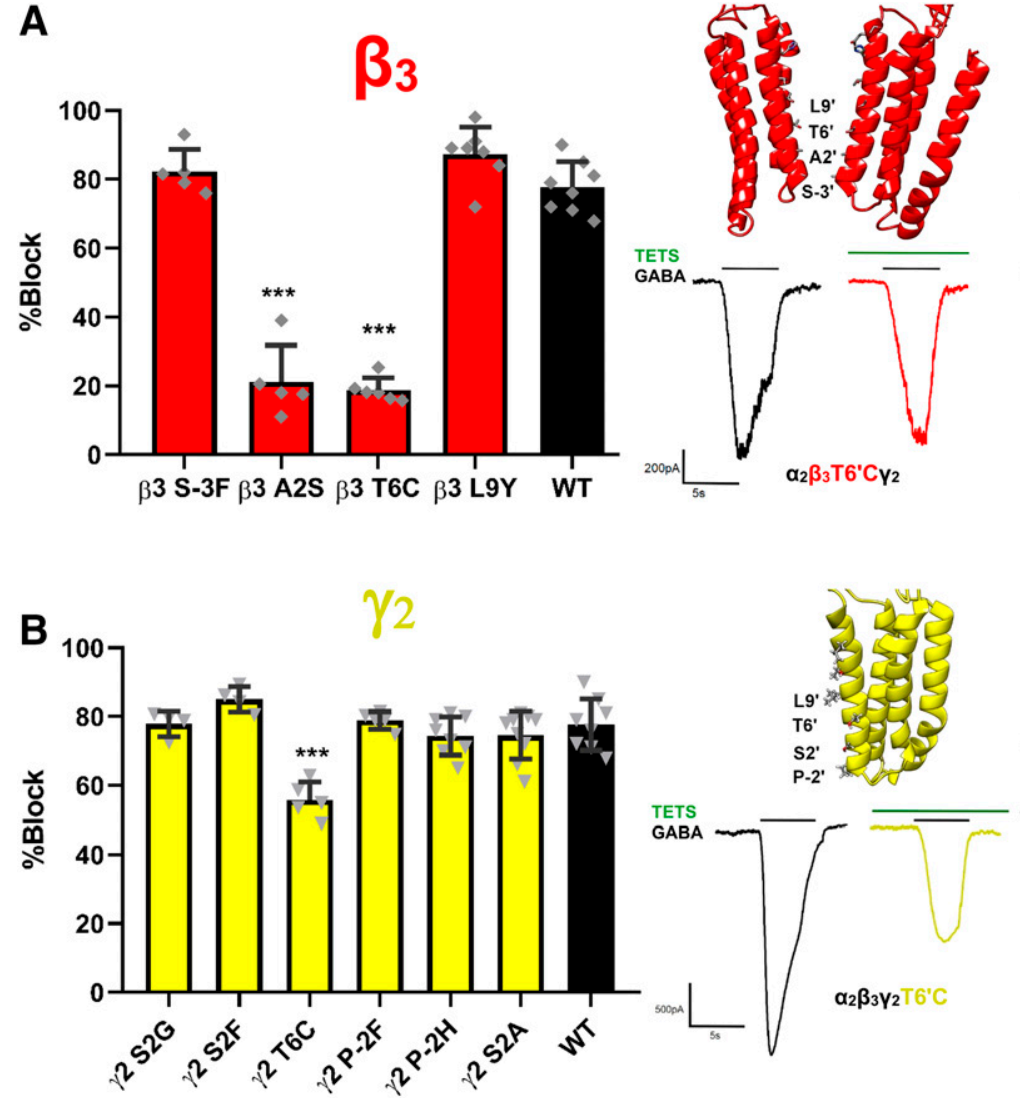

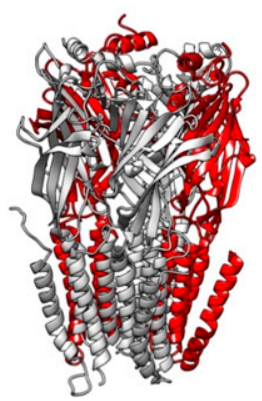

Fig. 4. Site-directed mutagenesis of the $\beta_{3}$ (A), $\gamma_{2}$ (B), and $\alpha_{2}$ (C) subunits of the $\alpha_{2} \beta_{3} \gamma_{2 \mathrm{~L}}$ receptor. Wild-type and mutant receptor combinations were recombinantly expressed in L929 cells. Scatter plots show the percentage of current inhibition obtained with $50 \mu \mathrm{M}$ of TETS when chloride currents were elicited by $\mathrm{EC}_{90}$ GABA $(100 \mu \mathrm{M})$. Cutaways of the homology model are shown next to the graphs to visualize the position of the mutated residues. A representative current trace from a T6' mutation is included for each subunit. Percentage of current blocked (mean \pm S.D. from $n=5$ to 8 cells per mutant) was analyzed with one-way ANOVA followed by Dunnett's test to compare the means with the WT control and to correct for multiple comparisons. * $P<0.05$; **, $P<0.01$; and $* * *, P<0.001$. WT, wild type.

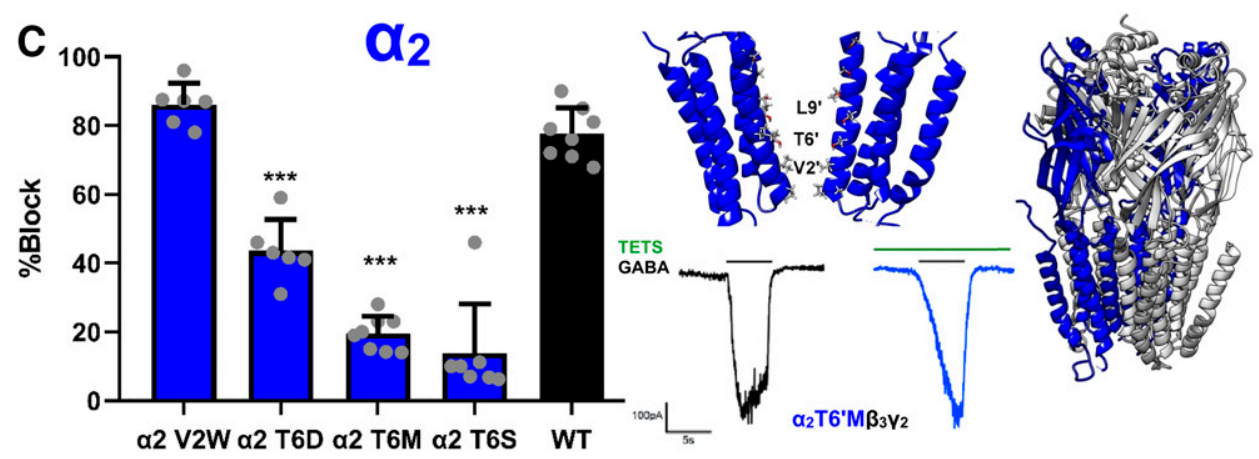

$\gamma_{2} \mathrm{~T}^{\prime}$. This made us suspect that the pore in this open-state model was too wide to optimally accommodate TETS. When the cryo-EM structure of the full-length human $\alpha_{1} \beta_{3} \gamma_{2 \mathrm{~L}}$ in lipid nanodiscs bound to picrotoxinin, bicuculline, alprazolam, and diazepam (Masiulis et al., 2019) was published, we therefore generated two new $\alpha_{2} \beta_{3} \gamma_{2}$ homology models based on the structures with bound alprazolam and GABA (pdb: 6HUO) and picrotoxinin (pdb: 6HUG).

The 6 HUO structure captured the $\alpha_{1} \beta_{3} \gamma_{2}$ receptor in a desensitized state that is closed down to $1.6 \AA$ at the $-2^{\prime}$ gate but open to $2.6 \AA$ at the activation-gate level at the $9^{\prime}$ Leu. When used as a template for a Rosetta-generated $\alpha_{2} \beta_{3} \gamma_{2}$ homology model, this structure produced a very similar binding model for TETS. RosettaLigand converged on a pose where the two sulfones of TETS are hydrogen bonding with the hydroxy groups of $\beta_{3} \mathrm{~T}^{\prime}$ and $\gamma_{2} \mathrm{~S} 2^{\prime}$, whereas one of the tertiary nitrogen atoms in the central cage of TETS accepts an additional hydrogen bond from $\gamma_{2} \mathrm{~T}^{\prime}$ (Supplemental Fig. 2).
We next probed a Rosetta-generated $\alpha_{2} \beta_{3} \gamma_{2}$ model based on the $6 \mathrm{HUG}$ template. In this template structure, the $\alpha_{1} \beta_{3} \gamma_{2 \mathrm{~L}}$ receptor has been captured at $3.1 \AA$ in what is assumed to be a closed/resting state (Masiulis et al., 2019). Picrotoxinin is positioned between the $2^{\prime}$ and $9^{\prime}$ rings with its lipophilic isopropenyl moiety surrounded by the L9' ring and its lactone rings forming putative hydrogen bonds with the $\mathrm{T} 6^{\prime}$ ring. The conduction pathway in this structure is "narrow" and constricted to $\sim 1.5 \AA$ at both the desensitization gate at $-2^{\prime}$ and the activation gate at $9^{\prime}$. We removed picrotoxinin from this structure, generated an $\alpha_{2} \beta_{3} \gamma_{2}$ model and then docked TETS into 50,000 random starting positions. The top scoring models in RosettaLigand showed a much "tighter" fit for TETS in this 6 HUG-based model than in the $\beta_{3}$ ECD $-\alpha_{5}$ TMD chimera-based open-state model or the 6HUO-based desensitized state model (Supplemental Fig. 3). When TETS is hydrogen bonding with $\beta_{3}$ and $\gamma_{2}$ at the $\mathrm{T}^{\prime}$ ring in the open-state model, there is a considerable amount of empty space between the TETS 
molecule and the other three $\mathrm{T} 6^{\prime}$ residues on the second $\beta_{3}$ and the two $\alpha_{2}$ subunits, which often made us wonder whether there could possibly be any water molecules participating in TETS binding at this position. However, in the narrower pore of the closed/resting-state model, TETS is very close to all five T6' residues (Supplemental Fig. 3). In the most frequently occurring low-energy binding pose, TETS accepts hydrogen bonds from the hydroxy groups of $\mathrm{T} 6$ ' from one of the two $\beta_{3}$ subunits and the $\gamma_{2}$ subunit as well as from the S2' on $\gamma_{2}$ (Fig. 5A). As previously, RosettaLigand again identified alternative poses in which the TETS has slightly turned and is now hydrogen bonding with one of its sulfone groups to one of the $\alpha_{2} \mathrm{~T} 6^{\prime}$ positions and the neighboring $\gamma_{2} \mathrm{~T} 6^{\prime}$ (Fig. 5B). But in both binding poses, the compact, "caged" TETS molecule shows perfect space complementarity and optimally fills out the pore lumen at the $\mathrm{T} 6^{\prime}$ ring of the pentameric receptor as shown in Fig. $5 \mathrm{C}$ when viewed from above. In addition to the three or two hydrogen bonds in the two alternative low-energy binding poses (Fig. 5, A and B), TETS is making a network of van der Waals contacts shown as black lines when viewed from the side (Fig. 5D) or from above looking down on the $\mathrm{T} 6^{\prime}$ ring (Fig. 5E). Supplemental Fig. 4 shows the dominant TETS binding pose in a 2D ligand-protein interaction diagram, which illustrates both hydrogen bonds and hydrophobic interactions together with the relative polarity of the interacting amino acid residues surrounding the TETS molecule. A comparison of the Rosetta energies for the three states showed the lowest energy for TETS binding in the closed/ resting-state model (-10.524 REU) versus -9.562 REU for the desensitized state and -4.955 REU for the open-state model. Because the Rosetta energy function is a combination of physics-based and statistics-based potentials, Rosetta energies are on an arbitrary scale referred to as REUs. Taken together, these results suggest that TETS is more likely to bind to the closed state of the $\alpha_{2} \beta_{3} \gamma_{2} \mathrm{GABA}_{\mathrm{A}}$ receptor than to the open state.

The T6' Ring Is Crucial for TETS Activity. We next more closely probed the 6HUG-based resting/closed-state model by obtaining full TETS concentration-response curves for selected mutants. Since RosettaLigand had consistently suggested two hydrogen bonds, multiple van der Waals interactions, and perfect space complementarity for TETS at the T6' ring, we first focused on this position. In keeping with there being two $\alpha_{2}$ and two $\beta_{3}$ subunits in the pentameric receptor, substituting $\mathrm{T} 6^{\prime}$ with a smaller but more hydrophobic cysteine or a larger and more hydrophobic methionine residue in these subunits drastically shifted the TETS concentration-response curve to the right and reduced the TETS $\mathrm{IC}_{50}$ by $\sim 700$ fold (Fig. $6 \mathrm{~A}$ ). In contrast, introducing a $\mathrm{T} 6^{\prime} \mathrm{C}$ mutation in the $\gamma_{2}$ subunit, which is only present once in the channel pentamer, had a less pronounced but still notable effect on TETS activity (Fig. 4B). Please note that we did not test TETS, which has an $\mathrm{LD}_{50}$ of $0.1 \mathrm{mg} / \mathrm{kg}$ and is an extremely potent convulsant, at concentrations higher than $500 \mu \mathrm{M}$ because of safety considerations. Coexpressing $\beta_{3} \mathrm{~T} 6^{\prime} \mathrm{C}$ and $\gamma_{2} \mathrm{~T} 6^{\prime} \mathrm{C}$ with wild-type $\alpha_{2}$ did not produce an additional shift in TETS sensitivity compared with the $\beta_{3} \mathrm{~T} 6^{\prime} \mathrm{C}$ mutation alone (unpublished data). Taken together, the $\mathrm{T} 6{ }^{\prime}$ mutation experiments demonstrate that the contacts at the $\mathrm{T} 6^{\prime}$ ring are essential for TETS activity. Introducing any residues that change the side-chain volume, charge, or hydropathy index in this position (see Fig. 4; Fig. 6A) is likely to disrupt the hydrogen bonds and van der Waals interactions that TETS is making and prevent TETS from accessing its optimal binding position.

We next turned our attention to the $2^{\prime}$ ring, where most models suggested a hydrogen bond (Fig. 3A; Fig. 5A) and van der Waals interactions (Fig. 5D) with the $\gamma_{2} \mathrm{~S} 2$ '. However, mutating this residue to $\mathrm{A}, \mathrm{G}$, or even $\mathrm{F}$ did not substantially reduce the blocking potency of $50 \mu \mathrm{M}$ TETS (Fig. 4B), and we therefore tried to do the opposite and increase the potency of TETS by creating an additional hydrogen bonding opportunity at the $2^{\prime}$ ring through the $\beta_{3} \mathrm{~A} 2$ 'S mutation. Interestingly, this mutation drastically reduced TETS potency by shifting the $\mathrm{IC}_{50}$ by 620 -fold to $300 \mu \mathrm{M}$ (Fig. 6B). In contrast, a closer look at the $\alpha_{2} \mathrm{~V} 2^{\prime} \mathrm{W}$ mutation with a full concentration-response curve revealed that this mutation, which introduces a large aromatic residue into the $2^{\prime}$ ring in two of the five subunits, affects TETS activity and increases the TETS $\mathrm{IC}_{50}$ from $480 \mathrm{nM}$ to $5.7 \mu \mathrm{M}$ (Fig. $6 \mathrm{~B}$ ).

TETS and Picrotoxinin Have Overlapping but Not Identical Binding Sites in the $\alpha_{2} \beta_{3} \gamma_{2} \mathbf{G A B A}_{\mathrm{A}}$ Receptor. We finally wondered how much the TETS and the picrotoxinin binding sites overlap in the $\alpha_{2} \beta_{3} \gamma_{2} \mathrm{GABA}_{\mathrm{A}}$ receptor and therefore docked picrotoxinin into our closed/ resting-state homology model. RosettaLigand converged on a dominant binding pose that was very similar to the cryoEM structure of the picrotoxinin binding site captured in the $\alpha_{1} \beta_{3} \gamma_{2 \mathrm{~L}}$ receptor (Masiulis et al., 2019). Supplemental Fig. 5 shows a comparison of the dominant picrotoxinin pose identified by RosettaLigand in the $\alpha_{2} \beta_{3} \gamma_{2}$ model and the picrotoxinin pose observed in the cryo-EM structure of the $\alpha_{1} \beta_{3} \gamma_{2 \mathrm{~L}}$ receptor. In both poses, the isopropenyl group is facing upwards toward the L9' ring, and its oxygen-rich lactone rings are centered at the $\mathrm{T} 6^{\prime}$ ring. However, in contrast to the cryo-EM structure, which suggested four putative hydrogen bonds for picrotoxinin in the $\alpha_{1} \beta_{3} \gamma_{2 \mathrm{~L}}$ receptor (two with the two $\beta_{3} \mathrm{~T}^{\prime}$ residues, one with $\alpha_{1} \mathrm{~T} 6^{\prime}$ and one with $\gamma_{2} \mathrm{~S}^{\prime}$ ), RosettaLigand only found three hydrogen bonds and suggested that picrotoxinin is turned and tilted a little differently in the $\alpha_{2} \beta_{3} \gamma_{2}$ homology model where it is hydrogen bonding with two $\beta_{3} \mathrm{~T} 6^{\prime}$ residues and $\gamma_{2} \mathrm{~T} 6^{\prime}$ but not $\alpha_{2}$ in the lowest-energy poses (Supplemental Fig. 4). Interestingly, this binding pose with fewer hydrogen bonds in the $\alpha_{2} \beta_{3} \gamma_{2}$ homology model than in the $\alpha_{1} \beta_{3} \gamma_{2}$ receptor structure is in line with picrotoxinin being less potent on $\alpha_{2} \beta_{3} \gamma_{2}\left(\mathrm{IC}_{50} 7.5 \mu \mathrm{M}\right)$ than on $\alpha_{1} \beta_{3} \gamma_{2}\left(\mathrm{IC}_{50} 3.7 \mu \mathrm{M}\right)$ in our hands when tested in patch-clamp experiments at $\mathrm{EC}_{90}$ GABA (Pressly et al., 2018).

An overlay of the dominant TETS and picrotoxinin binding poses in the $\alpha_{2} \beta_{3} \gamma_{2}$ receptor model (Fig. 7A) shows that both TETS and picrotoxinin are centered at the $\mathrm{T} 6^{\prime}$ ring but that the larger picrotoxinin extends further up into the L9' ring where the lipophilic isopropenyl group makes hydrophobic contacts with the lipophilic leucine residues as visualized in the space-filled renderings of picrotoxinin and TETS (Fig. 7A). When obtaining picrotoxinin concentration-response curves for the same two mutants $\left(\alpha_{2} \mathrm{~T} 6^{\prime} \mathrm{M}\right.$ and $\left.\beta_{3} \mathrm{~T} 6^{\prime} \mathrm{C}\right)$, which had practically made the $\alpha_{2} \beta_{3} \gamma_{2}$ receptor insensitive to TETS (Fig. 6A), we found that the $\alpha_{2}{ }^{\mathrm{T}} 6^{\prime} \mathrm{M}$ also rendered the $\alpha_{2} \beta_{3} \gamma_{2}$ receptor insensitive to picrotoxinin, whereas the $\beta_{3} \mathrm{~T}^{\prime} \mathrm{C}$ mutant remained as sensitive to picrotoxinin as the wildtype channel (Fig. 7B). Taken together, these modeling and 
A

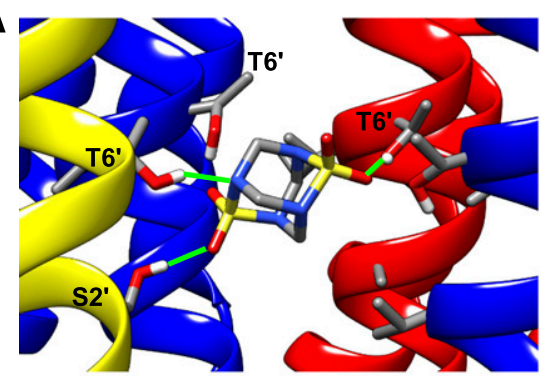

B

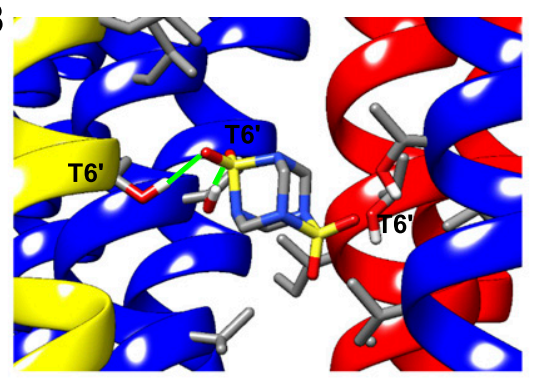

C

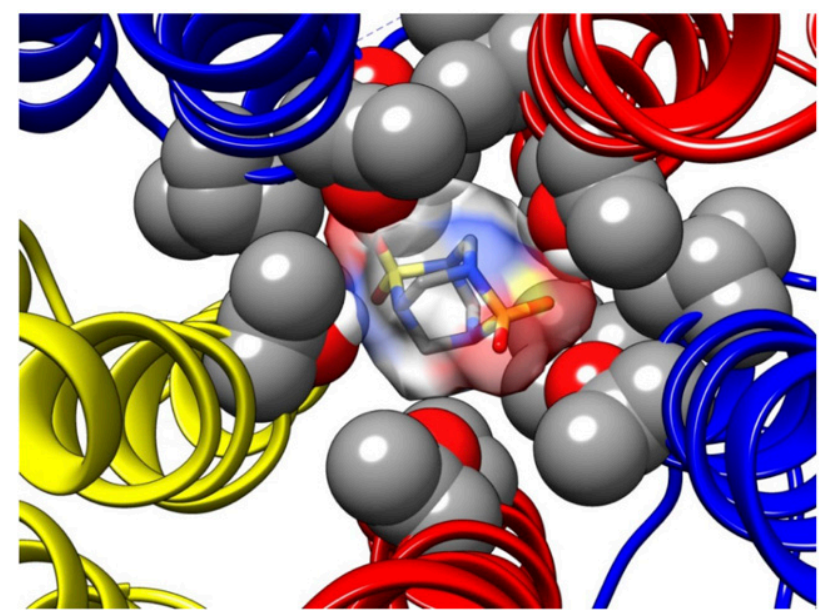

Fig. 5. TETS docked in the closed/resting-state homology model of the $\alpha_{2} \beta_{3} \gamma_{2} \mathrm{GABA}_{\mathrm{A}}$ receptor. (A) Transmembrane view of the dominant low-energy binding pose of TETS identified by RosettaLigand. (B) Alternative low-energy TETS binding pose. In both panels, one $\beta_{3}$ subunit has been removed for clarity. Hydrogen bonds are shown in green. The receptor is color-coded as follows: $\alpha_{2}$ (blue), $\beta_{3}$ (red), and $\gamma_{2}$ (yellow). (C) The TETS binding site viewed from above the $\mathrm{T} 6^{\prime}$ ring. TETS is shown in stick representation with a transparent molecular surface. The five threonine residues are rendered as spheres. (D) Van der Waals interactions of TETS shown in the same transmembrane view as in (A). (E) Van der Waals interactions of TETS viewed from above the $\mathrm{T} 6$ ' ring.
D

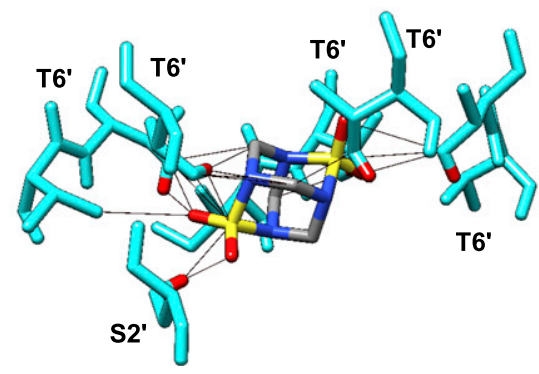

E

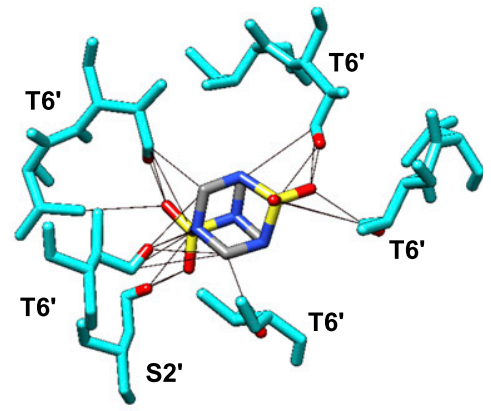

mutagenesis findings suggest that the picrotoxinin and the TETS binding sites are overlapping but not identical on $\alpha_{2} \beta_{3} \gamma_{2}$ $\mathrm{GABA}_{\mathrm{A}}$ receptors.

Confirmation of the Mutagenesis with a Chemical Biology Approach. To not solely rely on mutagenesis for identification the TETS binding site, we decided to confirm our results by altering the structure of the ligand instead of mutating the receptor site. Our group had previously synthesized a library of TETS derivatives for use as haptens for the development of an immunoassay for the detection of TETS (Barnych et al., 2017). We selected three of these TETS haptens (Fig. 8) and measured their ability to block chloride currents elicited by $100 \mu \mathrm{M}$ GABA $\left(\mathrm{EC}_{90}\right)$ through the $\alpha_{2} \beta_{3} \gamma_{2}$ $\mathrm{GABA}_{\mathrm{A}}$ receptor. Two TETS derivatives that enlarge (Hapten 2a) or open the tetraazaadamantane cage (Hapten $4 a$ ) were found to be roughly 15-fold less potent than TETS, whereas a derivative lacking one of the two hydrogen bond-accepting sulfone groups (Hapten 6b) was 180-fold less potent than TETS. To confirm that all three TETS haptens were still interacting with the $\mathrm{T}^{\prime}$ 'ring and that we were indeed investigating a structure-activity relationship, we also tested the three haptens at a concentration of $1 \mathrm{mM}$ on the $\beta_{3} \mathrm{~T} 6^{\prime} \mathrm{C}$ mutant (see gray box in Fig. 8). Although Hapten $2 \mathrm{a}$ and $4 \mathrm{a}$ still exhibited some effect that was dramatically reduced, Hapten $6 \mathrm{~b}$ was found to be ineffective on the $\beta_{3} \mathrm{~T} 6^{\prime} \mathrm{C}$ at $1 \mathrm{mM}$ (Fig. 8).

\section{Discussion}

We here used atomistic scale molecular modeling, site-directed mutagenesis, and whole-cell voltage-clamp experiments to map the binding site of the convulsant agent TETS in the $\alpha_{2} \beta_{3} \gamma_{2} \mathrm{GABA}_{\mathrm{A}}$ receptor. Like other "caged" convulsants, TETS interacts with residues in the M2 segment lining the chloride permeation pathway at the NCA site. That TETS binds to this site was suspected since the early 1990s when the Casida laboratory reported that TETS displaced $\left[{ }^{35} \mathrm{~S}\right]$ TBPS from what was then termed the mammalian-brain GABA-gated chloride channel in rat brain synaptosomes (Esser et al., 1991). However, since the first insect and mammalian $\mathrm{GABA}_{\mathrm{A}}$ receptors were cloned and recombinantly expressed, 
A

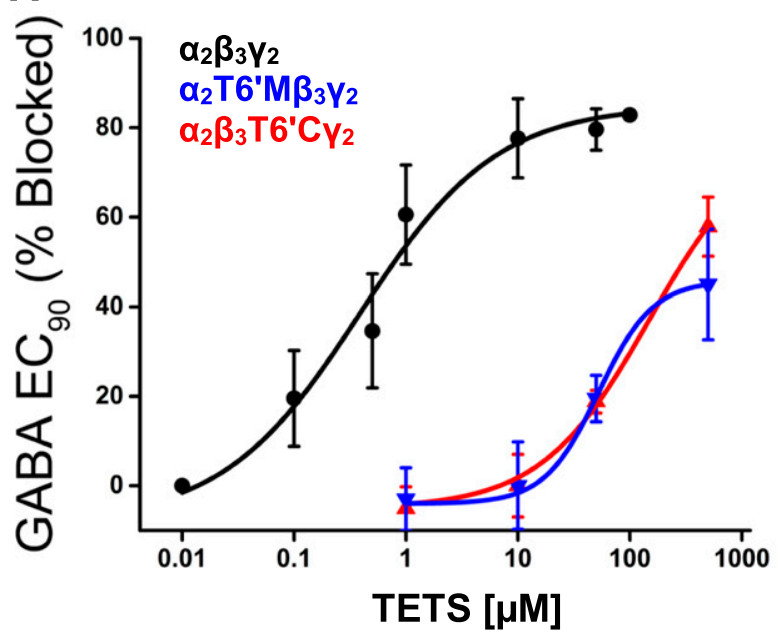

B

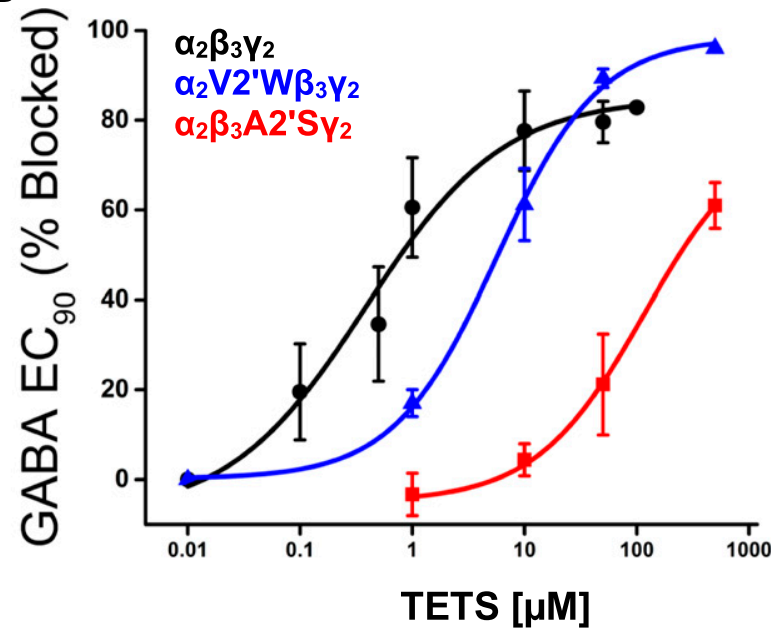

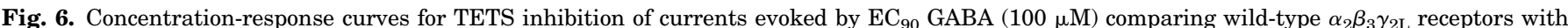

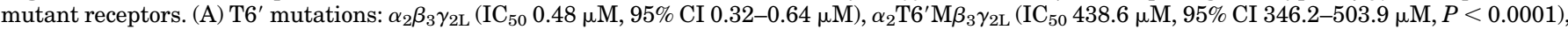

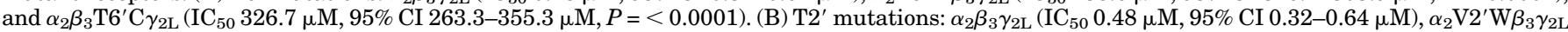

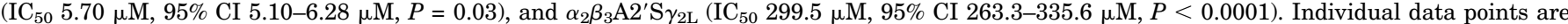

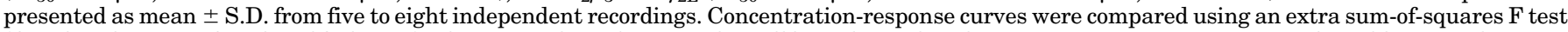

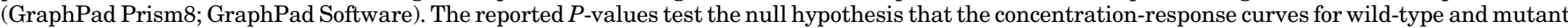
channels are identical. CI, confidence interval.

the question of the putative TETS binding site became somewhat confused. Although TETS was found to displace $\left[{ }^{3} \mathrm{H}\right]$ EBOB and $\left[{ }^{3} \mathrm{H}\right]$ dihydropicrotoxinin from Drosophila receptors or human $\alpha_{1} \beta_{3} \gamma_{2}$ receptors, it did not inhibit GABA-stimulated chloride fluxes through the human $\beta_{3}$ homopentamer (Ratra et al., 2001). To determine whether TETS indeed directly binds to $\mathrm{GABA}_{\mathrm{A}}$ receptors, the Casida group synthesized $\left[{ }^{14} \mathrm{C}\right]$ TETS in 2014 and used accelerated mass spectrometry to show that TETS can displace itself as well as $\left[{ }^{3} \mathrm{H}\right]$ EBOB and $\left[{ }^{35} \mathrm{~S}\right]$ TBPS from rat brain synaptosomes (Zhao et al., 2014). The authors of this study also generated a homology model of the $\alpha_{1} \beta_{2} \gamma_{2} \mathrm{GABA}_{\mathrm{A}}$ receptor based on the open-state structure of the Caenorhabditis elegans glutamate-gated chloride channel (Hibbs and Gouaux, 2011) and used molecular dynamics simulations to propose a putative TETS binding site in the lower pore of the $\alpha_{1} \beta_{2} \gamma_{2}$ $\mathrm{GABA}_{\mathrm{A}}$ receptor where TETS was predicted to make three hydrogen-bonding interactions with two $\alpha 1 \mathrm{~T} 1^{\prime}$ residues and $\gamma 2 \mathrm{~S} 2 '$. However, although appearing reasonable at the time, this prediction, which was never confirmed by mutagenesis, was called into question when the subsequently solved $\mathrm{GABA}_{\mathrm{A}}$ receptor structures showed that $\mathrm{T} 1^{\prime}$ was not a pore-lining residue and that $\mathrm{GABA}_{\mathrm{A}}$ receptors in various states are narrower at 2' (Miller et al., 2017; Laverty et al., 2019; Masiulis et al., 2019) and not splayed open as widely as the C. elegans $\mathrm{GluCl}$ channel, making it impossible for TETS to hydrogen bond with $\mathrm{T} 1$ '.

When determining the $\mathrm{GABA}_{\mathrm{A}}$ receptor subtype selectivity of TETS, we observed that TETS inhibits $\alpha_{2} \beta_{3} \gamma_{2}$ and $\alpha_{6} \beta_{3} \gamma_{2}$ $\mathrm{GABA}_{\mathrm{A}}$ receptors with submicromolar $\mathrm{IC}_{50} \mathrm{~s}$ in whole-cell patch-clamp experiments while it blocks $\alpha_{1}, \alpha_{4}, \beta_{2}, \gamma_{1}$, or $\delta$ containing $\mathrm{GABA}_{\mathrm{A}}$ receptors roughly 5-10-fold less potently and basically has no effect on $\beta_{1}$-containing receptor combinations (Pressly et al., 2018), suggesting a preference for the $\alpha_{2}$ or $\alpha_{6} / \beta_{3} / \gamma_{2}$ combination. We therefore here set out to map the binding site of TETS on the $\alpha_{2} \beta_{3} \gamma_{2}$ receptor, which we believe is the pharmacologically most-relevant
$\mathrm{GABA}_{\mathrm{A}}$ receptor for the seizure-inducing activity of TETS. Since we had previously successfully used the Rosetta molecular modeling suite to make a homology model of the pore region of the calcium-activated potassium channel KCa3.1 (Nguyen et al., 2017) that agreed well with the subsequently solved cryo-EM structure (Lee and MacKinnon, 2018) and that allowed us to correctly predict the binding sites of several KCa3.1 inhibitors in either the pore or the fenestration region of KCa3.1 with RosettaLigand, we again used the Rosetta Membrane method (Rohl et al., 2004; Yarov-Yarovoy et al., 2012) for homology modeling of the $\alpha_{2} \beta_{3} \gamma_{2}$ receptor but this time in combination with the more recently developed RosettaES refinement approach (Frenz et al., 2017), which is particularly useful for "cleaning up" and relaxing cryo-EM structures before docking ligands. When we started this work, the structure that seemed most attractive to us as a template was the X-ray structure of a chimeric channel in which the ECD of the human $\beta_{3}$ subunit had been fused to the TMD of the human $\alpha_{5}$ subunit (Miller et al., 2017). Although the ECD of this chimera, which can only be activated by histamine and not by the natural ligand GABA because it is lacking the GABA binding site in the $\alpha / \beta$ interface, might be of questionable value for modeling the ECD, the membrane-embedded part of this chimera was appealing because its pore showed two constriction sites at the intracellular end at the $2^{\prime}$ and $-2^{\prime}$ ring, and binding of the positive allosteric modulator pregnanolone enlarged the pore diameter at the -2 proline ring. The $\beta_{3}$ ECD- $\alpha_{5}$ TMD chimera was further capable of producing picrotoxin-sensitive chloride currents (Miller et al., 2017), suggesting that it contained an NCA site in the TMD. After generating what we here assume to be an open-state homology model of the $\alpha_{2} \beta_{3} \gamma_{2}$ receptor using Rosetta (Fig. 3A), we first probed the putative NCA site of our model by docking picrotoxinin and EBOB. For both these "classical" antagonists, RosettaLigand identified binding poses that were in good agreement with literature and existing mutagenesis data (Esser et al., 1991; Xu et al., 1995; Chen et al., 2006; Erkkila 

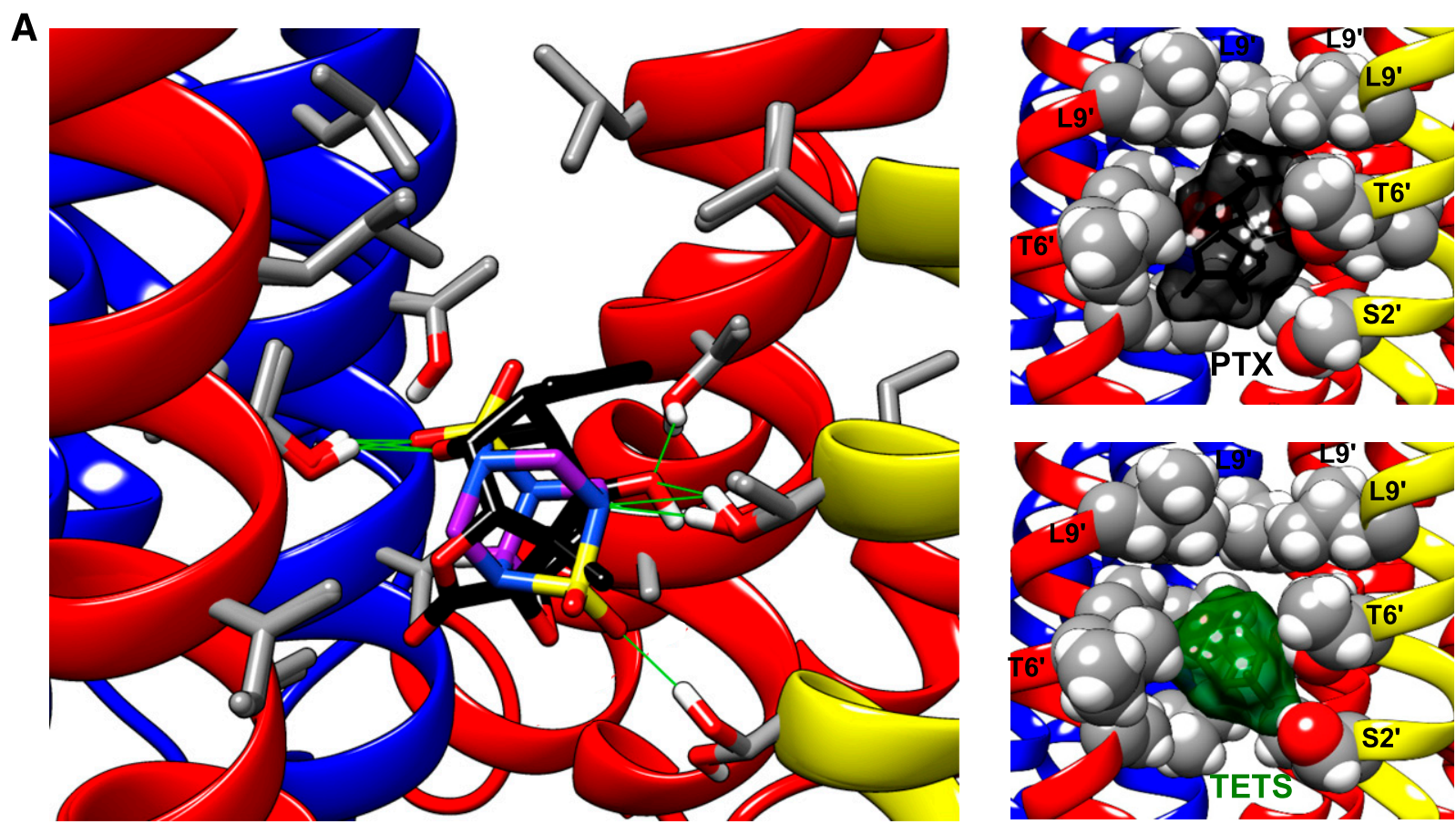

B

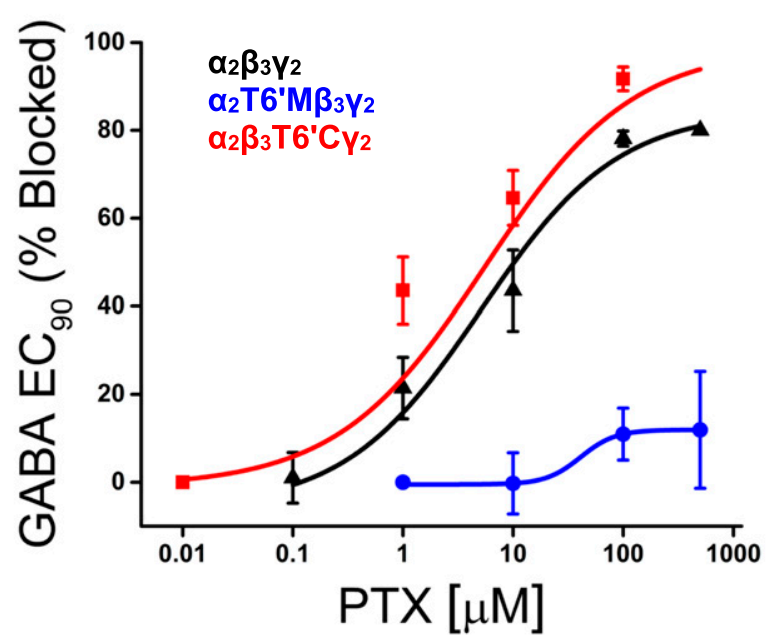

Fig. 7. (A) Overlay of the lowest-energy binding poses of TETS and picrotoxinin in stick representation in the closed/resting-state homology model of the $\alpha_{2} \beta_{3} \gamma_{2}$ GABA $_{\mathrm{A}}$ receptor. The receptor is color-coded as follows: $\alpha_{2}$ (blue), $\beta_{3}$ (red), and $\gamma_{2}$ (yellow). One $\alpha_{2}$ subunit is removed for clarity. Picrotoxinin is shown in black. Hydrogen bonds are shown in green. The panels on the side show transparent molecular surfaces of picrotoxinin (black) and TETS (green) with pore-lining resides in the $\mathrm{L9}^{\prime}, \mathrm{T} 6^{\prime}$, and $2^{\prime}$ ring rendered as spheres. (B) Concentration-response curves for picrotoxinin inhibition of currents evoked by $\mathrm{EC}_{90} \mathrm{GABA}(100 \mu \mathrm{M})$ comparing wild-type $\alpha_{2} \beta_{3} \gamma_{2 \mathrm{~L}}$ receptors with T6' mutations: $\alpha_{2} \beta_{3} \gamma_{2 \mathrm{~L}}$ (IC 50 6.8 $\left.\mu \mathrm{M}, 95 \% \mathrm{CI} 4.5-8.4 \mu \mathrm{M}\right)$, $\alpha_{2} \mathrm{~T} 6^{\prime} \mathrm{M} \beta_{3} \gamma_{2 \mathrm{~L}}$ (no meaningful IC $\mathrm{IC}_{50}$ can be determined since the maximal effect is drastically reduced, and we therefore consider this channel insensitive to picrotoxinin), and $\alpha_{2} \beta_{3} \mathrm{~T} 6^{\prime} \mathrm{C} \gamma_{2 \mathrm{~L}}\left(\mathrm{IC}_{50} 4.2 \mu \mathrm{M}, 95 \% \mathrm{CI} 1.2-6.9 \mu \mathrm{M}, P=0.2\right.$ ). Individual data points are presented as mean \pm S.D. from five to eight independent recordings. Concentration-response curves were compared using an extra sum-of-squares F test (GraphPad Prism8; GraphPad Software). The reported $P$-values test the null hypothesis that the concentration-response curves for wild-type and mutant channels are identical. CI, confidence interval.

et al., 2008; Ng et al., 2016). Encouraged by this validation, we next used three different ligand-docking algorithms, RosettaLigand, Glide, and Swissdock, with different energy functions, different parametrizations, and different requirements for initial ligand placements to search for the TETS binding site (Fig. 3). Specifically, although RosettaLigand and Glide require ligands to be placed into predefined boxes for which the diameter can be chosen, which somewhat biases the search toward potential binding sites selected by the user, Swissdock requires no such assumption. The different programs found two possible binding sites for TETS: one site at the $\mathrm{T} 6^{\prime}$ ring, which was identified by all three programs, and a second site lower down at the entrance to the permeation pathway. Sitedirected mutagenesis experiments (Fig. 4) ruled out the lower site and confirmed that TETS interacts with the T6' ring where threonine residues from all five subunits line the channel pore.

Although the predominant TETS binding poses identified by RosettaLigand at the $\mathrm{T} 6^{\prime}$ ring of our open-state model agreed with the mutagenesis, TETS was only partially 
<smiles>O=S1(=O)N2CN(CN1C(F)(F)F)N(S(=O)(=O)C(F)(F)F)C2</smiles><smiles>CS(=O)(=O)N1CN2CCN(C2)S1(=O)=O</smiles>

Hapten 2a<smiles>NC12CN3CN(CN(C3)S1(=O)=O)C2</smiles>

Hapten 6b<smiles>CN1CN2CN(S(=O)(=O)N3CCCC3)S(=O)(=O)C12</smiles>

Hapten 4a
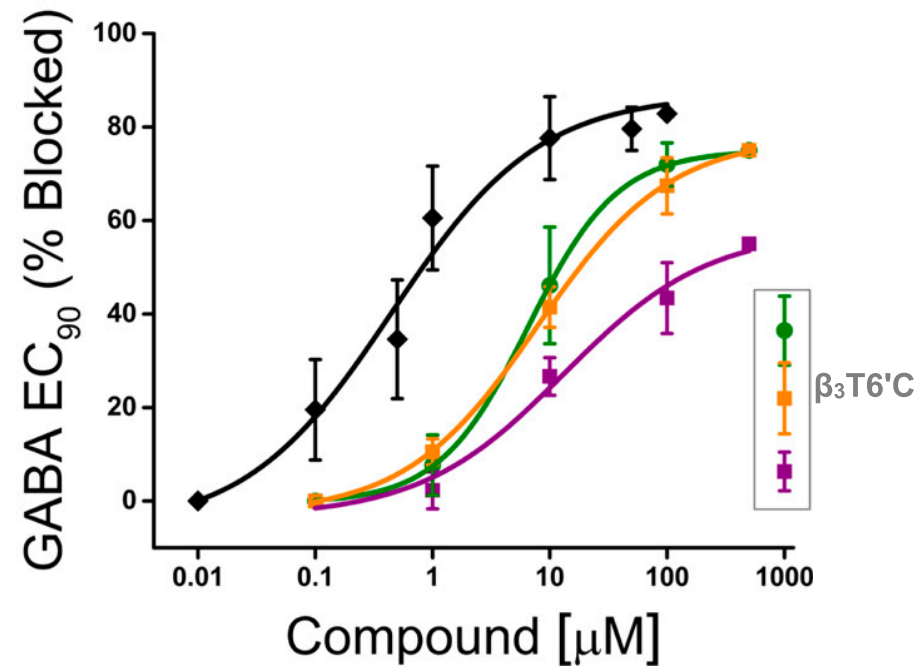

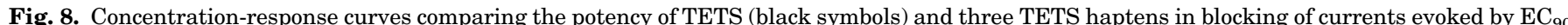

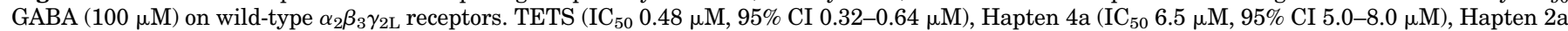

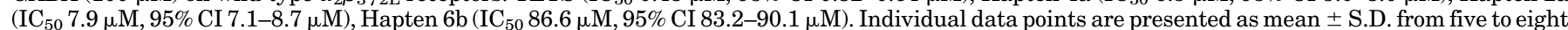

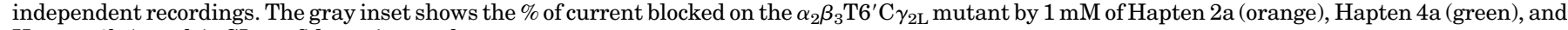
Hapten 6b (purple). CI, confidence interval

occluding the pore (Supplemental Fig. 3), raising the question of why this position was so sensitive to relatively small changes in side-chain volume in the mutagenesis and making us wonder whether we could be missing water molecules, which are not explicitly modeled by Rosetta. Fortunately, while we were working on this project, the cryo-EM structure of the human $\alpha_{1} \beta_{3} \gamma_{2}$ receptor in a lipid environment was published in both the closed and the desensitized states (Laverty et al., 2019; Masiulis et al., 2019). Using these new templates, we generated two additional homology models of the $\alpha_{2} \beta_{3} \gamma_{2}$ receptor in the desensitized and closed/resting states and again docked TETS. Although the docking poses in the desensitized state somewhat reduced the space at the $\mathrm{T} 6^{\prime}$ ring (Supplemental Fig. 3), the dominant docking poses of TETS in the closed/resting model had the comparatively lowest Rosetta energy and showed a much "tighter" fit for TETS (Fig. 5), which now perfectly "snuggled" into the T6" ring by making three hydrogen bonds and literally a net of van der Waals interactions in its dominant low-energy binding pose (Fig. 5). The perfect space complementarity and the multitude of interactions observed in this pose also provided a much better explanation for why the introduction of any residue that changes side-chain volume, charge, or hydropathy index in the $\mathrm{T}^{\prime}{ }^{\prime}$ ring reduces TETS activity (Fig. 4; Fig. $6 \mathrm{~A}$ ). In contrast, the results of mutating the $2^{\prime}$ ring, where TETS is again seen to hydrogen bond with $\gamma_{2} \mathrm{~S} 2^{\prime}$ in our closed/resting-state model, were not as straightforward to interpret. Introducing a large, aromatic $\operatorname{Trp}$ in $\alpha_{2}$ shifted TETS potency, as would be expected for introducing steric bulk into the $2^{\prime}$ ring, but mutations of the $\gamma_{2} \mathrm{~S} 2$ ' residue itself did not have the expected effects. However, when we then replaced Ala with Ser in $\beta_{3}$ in an attempt to potentially pick up an additional hydrogen bonds, we saw a dramatic reduction in TETS potency (Fig. 6B). One explanation for this finding could be that maybe we did indeed create an additional hydrogen-bonding opportunity and were now "catching" TETS at the $2^{\prime}$ ring and preventing it from accessing the $\mathrm{T} 6^{\prime}$ ring. Interestingly, our finding that an A2'S mutation reduces TETS potency $~ 600$-fold is in line with reports that in the insect RDL receptor, the A2'S mutation confers resistance to cyclodiene insecticides and picrotoxin (Ffrench-Constant et al., 1993; Zhang et al., 1994) and reduces fipronil activity (Zhang et al., 2016).

Based on our molecular modeling results, which showed a "tighter" fit and a relatively lower energy in a resting/closed state than in an open or desensitized state model of the $\alpha_{2} \beta_{3} \gamma_{2}$ receptor, we suggest that TETS preferentially binds to the closed state. This assumption is in line with our observations that in electrophysiological experiments current inhibition by TETS shows no delays and that TETS produces more block if $\mathrm{GABA}_{\mathrm{A}}$ receptors are preincubated with TETS before channels are activated with GABA (Fig. 1A) than if GABA and TETS are applied simultaneously (Fig. 1B). The template for our closed-state model was the cryo-EM structure of the $\alpha_{1} \beta_{3} \gamma_{2 \mathrm{~L}}$ receptor (Masiulis et al., 2019) in complex with picrotoxinin (pdb:6HUG), which, based on the pore radius at the $-2^{\prime}$ and $9^{\prime}$, is closed at both the desensitization and the activation gate and therefore assumed to have captured the channel in the closed/resting state. Picrotoxinin, which has been reported to be an open-channel blocker while also sometimes displaying some competitive inhibitor effects $(\mathrm{Xu}$ et al., 1995; Mehta and Ticku, 2001; Wang et al., 2006; Olsen et al., 2019), is believed to bind initially to an open-channel pore and then to stabilize a closed/resting receptor state (Masiulis et al., 2019). We here show that the TETS and the picrotoxinin binding sites in the $\alpha_{2} \beta_{3} \gamma_{2}$ receptor are overlapping but not identical. TETS is centered at the $\mathrm{T} 6^{\prime}$ ring with an additional contact at the $2^{\prime}$ ring, whereas the larger picrotoxinin extends further up into the pore to the $L 9^{\prime}$ ring (Fig. 7). It therefore is possible that TETS differs from picrotoxinin, and it would be desirable in the future to determine its structure in complex with the $\alpha_{2} \beta_{3} \gamma_{2}$ receptor. TETS could be binding directly to the closed state or like picrotoxinin initially interact with the open state, enhance the rate of current decay, and then stabilize the closed state. 


\section{Acknowledgments}

We would like to thank Vladimir Yarov-Yarovoy for help with Rosetta and gratefully acknowledge use of OMEGA version 2.5.1.4 (OpenEye Scientific Software, Santa Fe, NM; http://www.eyesopen.com).

\section{Authorship Contributions}

Participated in research design: Pressly, Lee, Wulff.

Conducted experiments: Pressly, Lee.

Contributed new reagents or analytic tools: Barnych, Hammock.

Performed data analysis: Pressly.

Wrote or contributed to the writing of the manuscript: Pressly, Lee, Barnych, Hammock, Wulff.

\section{References}

Barnych B, Vasylieva N, Joseph T, Hulsizer S, Nguyen HM, Cajka T, Pessah I, Wulff H, Gee SJ, and Hammock BD (2017) Development of Tetramethylendisulfotetramine (TETS) hapten library: synthesis, electrophysiological studies, and immune response in rabbits. Chemistry 23:8466-8472.

Casida JE and Durkin KA (2015) Novel GABA receptor pesticide targets. Pestic Biochem Physiol 121:22-30.

Chen L, Durkin KA, and Casida JE (2006) Structural model for gamma-aminobutyric acid receptor noncompetitive antagonist binding: widely diverse structures fit the same site. Proc Natl Acad Sci USA 103:5185-5190.

Davis IW and Baker D (2009) RosettaLigand docking with full ligand and receptor flexibility. J Mol Biol 385:381-392.

Erkkila BE, Sedelnikova AV, and Weiss DS (2008) Stoichiometric pore mutations of the GABAAR reveal a pattern of hydrogen bonding with picrotoxin. Biophys $J \mathbf{9 4}$ $4299-4306$

Esser T, Karu AE, Toia RF, and Casida JE (1991) Recognition of tetramethylenedisulfotetramine and related sulfamides by the brain GABA-gated chloride channel and a cyclodiene-sensitive monoclonal antibody. Chem Res Toxicol 4:162-167.

Ffrench-Constant RH, Rocheleau TA, Steichen JC, and Chalmers AE (1993) A point mutation in a Drosophila GABA receptor confers insecticide resistance. Nature 363:449-451.

Frenz B, Walls AC, Egelman EH, Veesler D, and DiMaio F (2017) RosettaES a sampling strategy enabling automated interpretation of difficult cryo-EM maps. Nat Methods 14:797-800.

García-Nafría J and Tate CG (2020) Cryo-Electron microscopy: moving beyond X-ray crystal structures for drug receptors and drug development. Annu Rev Pharmacol Toxicol 60:51-71.

Haskell AR and Voss E (1957) The pharmacology of tetramine (tetraethylenedisulfotetramine). J Am Pharm Assoc Am Pharm Assoc 46:239-242.

Hawkins PC and Nicholls A (2012) Conformer generation with OMEGA: learning from the data set and the analysis of failures. J Chem Inf Model 52:2919-2936.

Hawkins PC, Skillman AG, Warren GL, Ellingson BA, and Stahl MT (2010) Conformer generation with OMEGA: algorithm and validation using high quality structures from the Protein Databank and Cambridge Structural Database. $J$ Chem Inf Model 50:572-584.

Hibbs RE and Gouaux E (2011) Principles of activation and permeation in an anionselective Cys-loop receptor. Nature 474:54-60.

Jenkins DP, Strøbæk D, Hougaard C, Jensen ML, Hummel R, Sørensen US, Christophersen $\mathrm{P}$, and Wulff $\mathrm{H}(2011)$ Negative gating modulation by $(R)-N$-(benzimidazol-2-yl)-1,2,3,4-tetrahydro-1-naphthylamine (NS8593) depends on residues in the inner pore vestibule: pharmacological evidence of deep-pore gating of $\mathrm{K}(\mathrm{Ca}) 2$ channels. Mol Pharmacol 79:899-909.

Krall J, Balle T, Krogsgaard-Larsen N, Sørensen TE, Krogsgaard-Larsen P, Kristiansen U, and Frølund $\mathrm{B}$ (2015) $\mathrm{GABA}_{\mathrm{A}}$ receptor partial agonists and antagonists: structure, binding mode, and pharmacology. Adv Pharmacol 72:201-227.

Lauková M, Velíšková J, Velíšek L, and Shakarjian MP (2020) Tetramethylenedisulfotetramine neurotoxicity: what have we learned in the past 70 years? Neurobiol Dis 133:104491.

Laverty D, Desai R, Uchański T, Masiulis S, Stec WJ, Malinauskas T, Zivanov J, Pardon E, Steyaert J, Miller KW, et al. (2019) Cryo-EM structure of the human $\alpha 1 \beta 3 \gamma 2 \mathrm{GABA}_{\mathrm{A}}$ receptor in a lipid bilayer. Nature 565:516-520.

Lee CH and MacKinnon R (2018) Activation mechanism of a human SK-calmodulin channel complex elucidated by cryo-EM structures. Science 360:508-513.

Masiulis S, Desai R, Uchański T, Serna Martin I, Laverty D, Karia D, Malinauskas T, Zivanov J, Pardon E, Kotecha A, et al. (2019) GABA $_{A}$ receptor signalling mechanisms revealed by structural pharmacology [published correction appears in $\mathrm{Na}$ ture (2019) 566:E8]. Nature 565:454-459.
Mehta AK and Ticku MK (2001) Characterization of the picrotoxin site of GABAA receptors. Curr Protoc Pharmacol Chapter 1:Unit 1.18.

Meiler J and Baker D (2006) ROSETTALIGAND: protein-small molecule docking with full side-chain flexibility. Proteins 65:538-548.

Miller PS and Aricescu AR (2014) Crystal structure of a human GABA $_{\mathrm{A}}$ receptor. Nature 512:270-275.

Miller PS, Scott S, Masiulis S, De Colibus L, Pardon E, Steyaert J, and Aricescu AR (2017) Structural basis for $\mathrm{GABA}_{\mathrm{A}}$ receptor potentiation by neurosteroids. Nat Struct Mol Biol 24:986-992.

Ng CC, Duke RK, Hinton T, and Johnston GA (2016) GABA $_{A}$ receptor cysteinyl mutants and the ginkgo terpenoid lactones bilobalide and ginkgolides. Eur $J$ Pharmacol 777:136-146.

Nguyen HM, Singh V, Pressly B, Jenkins DP, Wulff H, and Yarov-Yarovoy V (2017) Structural insights into the atomistic mechanisms of action of small molecule inhibitors targeting the KCa3.1 channel pore. Mol Pharmacol 91:392-402.

Olsen RW (2006) Picrotoxin-like channel blockers of GABA A $_{\text {receptors. Proc Natl }}$ Acad Sci USA 103:6081-6082.

Olsen RW (2015) Allosteric ligands and their binding sites define $\gamma$-aminobutyric acid (GABA) type A receptor subtypes. Adv Pharmacol 73:167-202.

Olsen RW, Lindemeyer AK, Wallner M, Li X, Huynh KW, and Zhou ZH (2019) Cryo-electron microscopy reveals informative details of $\mathrm{GABA}_{\mathrm{A}}$ receptor structural pharmacology: implications for drug discovery. Ann Transl Med 7 (Suppl 3):S144

Olsen RW and Sieghart W (2008) International Union of Pharmacology. LXX Subtypes of gamma-aminobutyric acid(A) receptors: classification on the basis of subunit composition, pharmacology, and function. Update. Pharmacol Rev 60: $243-260$

Othman NA, Gallacher M, Deeb TZ, Baptista-Hon DT, Perry DC, and Hales TG (2012) Influences on blockade by t-butylbicyclo-phosphoro-thionate of GABA(A) receptor spontaneous gating, agonist activation and desensitization. J Physiol 590:163-178.

Pettersen EF, Goddard TD, Huang CC, Couch GS, Greenblatt DM, Meng EC, and Ferrin TE (2004) UCSF Chimera--a visualization system for exploratory research and analysis. J Comput Chem 25:1605-1612.

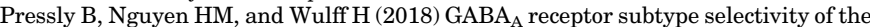
proconvulsant rodenticide TETS. Arch Toxicol 92:833-844.

Pressly B, Vasylieva N, Barnych B, Singh V, Singh L, Bruun DA, Hwang SH, Chen YJ, Fettinger JC, Johnnides S, et al. (2020) Comparison of the toxicokinetics of the convulsants picrotoxinin and tetramethylenedisulfotetramine (TETS) in mice. Arch Toxicol 94:1995-2007.

Ratra GS, Kamita SG, and Casida JE (2001) Role of human GABA(A) receptor beta3 subunit in insecticide toxicity. Toxicol Appl Pharmacol 172:233-240.

Rohl CA, Strauss CE, Misura KM, and Baker D (2004) Protein structure prediction using Rosetta. Methods Enzymol 383:66-93.

Wang DS, Mangin JM, Moonen G, Rigo JM, and Legendre P (2006) Mechanisms for picrotoxin block of alpha2 homomeric glycine receptors. J Biol Chem 281: 3841-3855.

Wulff H, Gutman GA, Cahalan MD, and Chandy KG (2001) Delineation of the clotrimazole/TRAM-34 binding site on the intermediate conductance calcium-activated potassium channel, IKCa1. J Biol Chem 276:32040-32045.

Xu M, Covey DF, and Akabas MH (1995) Interaction of picrotoxin with GABAA receptor channel-lining residues probed in cysteine mutants. Biophys $J \mathbf{6 9}$ : 1858-1867.

Yarov-Yarovoy V, DeCaen PG, Westenbroek RE, Pan CY, Scheuer T, Baker D, and Catterall WA (2012) Structural basis for gating charge movement in the voltage sensor of a sodium channel. Proc Natl Acad Sci USA 109:E93-E102.

Zhang HG, ffrench-Constant RH, and Jackson MB (1994) A unique amino acid of the Drosophila GABA receptor with influence on drug sensitivity by two mechanisms. J Physiol 479:65-75.

Zhang Y, Meng X, Yang Y, Li H, Wang X, Yang B, Zhang J, Li C, Millar NS, and Liu Z (2016) Synergistic and compensatory effects of two point mutations conferring target-site resistance to fipronil in the insect GABA receptor RDL. Sci Rep 6:32335.

Zhao C, Hwang SH, Buchholz BA, Carpenter TS, Lightstone FC, Yang J, Hammock BD, and Casida JE (2014) GABAA receptor target of tetramethylenedisulfotetramine [published correction appears in Proc Natl Acad Sci U S A (2014) 111:11223]. Proc Natl Acad Sci USA 111:8607-8612.

Zimin PI, Garic B, Bodendiek SB, Mahieux C, Wulff H, and Zhorov BS (2010) Potassium channel block by a tripartite complex of two cationophilic ligands and a potassium ion. Mol Pharmacol 78:588-599.

Address correspondence to: Heike Wulff, Department of Pharmacology, Genome and Biomedical Sciences Facility, Room 3502, 451 Health Sciences Dr., University of California, Davis, CA 95616. E-mail: hwulff@ucdavis.edu 